\title{
STRUCTURE OF HOCHSCHILD COHOMOLOGY OF PATH ALGEBRAS AND DIFFERENTIAL FORMULATION OF EULER'S POLYHEDRON FORMULA*
}

\author{
LI GUO ${ }^{\dagger}$ AND FANG $\mathrm{LI}^{\ddagger}$
}

\begin{abstract}
This article studies the Lie algebra $\operatorname{Der}(\mathbf{k} \Gamma)$ of derivations on the path algebra $\mathbf{k} \Gamma$ of a quiver $\Gamma$ and the Lie algebra on the first Hochschild cohomology group $H H^{1}(\mathbf{k} \Gamma)$. We relate these Lie algebras to the algebraic and combinatorial properties of the path algebra. Characterizations of derivations on a path algebra are obtained, leading to a canonical basis of $\operatorname{Der}(\mathbf{k} \Gamma)$ and its Lie algebra properties. Special derivations are associated to the vertices, arrows and faces of a quiver, and the concepts of a connection matrix and boundary matrix are introduced to study the relations among these derivations, giving rise to an interpretation of Euler's polyhedron formula in terms of derivations. By taking dimensions, this relation among spaces of derivations recovers Euler's polyhedron formula. This relation also leads to a combinatorial construction of a canonical basis of the Lie algebra $H H^{1}(\mathbf{k} \Gamma)$, together with a new semidirect sum decomposition of $H H^{1}(\mathbf{k} \Gamma)$.
\end{abstract}

Key words. quiver, path algebra, Hochschild cohomology, Lie algebra, differential algebra, graph, Euler's polyhedron formula, connection matrix.

AMS subject classifications. 16E40, 16G20, 05E15, 05C25, 12H05, 16W25, $16 \mathrm{~S} 32$.

1. Introduction. This paper studies the structure of the Lie algebra of derivations on the path algebra $\mathbf{k} \Gamma$ of a quiver $\Gamma$ and the Lie algebra of outer derivations on the path algebra, also known as the first Hochschild cohomology group $H H^{1}(\mathbf{k} \Gamma)$. This study has two motivations, one from Hochschild cohomology and one from differential algebra. We determine a canonical basis and their multiplication constants for these two Lie algebras, and relate it to the combinatorial properties of the quiver, such as Euler's Polyhedron Theorem.

The study of Hochschild cohomology of quiver related algebras started with the dimension formula for $H H^{n}(\mathbf{k} \Gamma)$ given by Happel in 1989 [11], who showed that for an acyclic quiver $\Gamma$ and a field $\mathbf{k}$,

$$
H H^{0}(\mathbf{k} \Gamma)=\mathbf{k}, \quad \operatorname{dim}_{\mathbf{k}} H H^{1}(\mathbf{k} \Gamma)=1-|V|+\sum_{\alpha \in E} v(\alpha), \quad H H^{i}(\mathbf{k} \Gamma)=0, \forall i \geq 2
$$

where $v(\alpha)=\operatorname{dim}_{\mathbf{k}} t(\alpha) \mathbf{k} \Gamma h(\alpha)$, while $V$ and $E$ are respectively the sets of vertices and arrows of $\Gamma$. Afterwards, there have been extensive studies on the dimensions of the Hochschild cohomology groups of quiver related algebras, such as truncated path algebras, monomial algebras, schurian algebras and 2-nilpotent algebras $[1,6,11,19$, $20,23,24,26,29]$. In [24], the first Hochschild cohomology group of a monomial algebra of radical square zero is given as the splitting extension of a semi-simple Lie algebra by the radical due to Levi-Malcev Theorem where the semisimple Lie algebra is related to the cardinality of parallel arrows and the radical to the Euler characteristic of the quiver. The quiver is supposed to be planar.

Further understanding of the Lie algebra $H H^{1}(\mathbf{k} \Gamma)$ would benefit from an explicit structure of this Lie algebra, such as a canonical basis and the corresponding multiplication constants. This is what we achieve in this paper. We find that the choice

\footnotetext{
*Received August 23, 2012; accepted for publication April 3, 2013.

${ }^{\dagger}$ Department of Mathematics and Computer Science, Rutgers University, Newark, NJ 07102, USA (liguo@rutgers.edu).

${ }^{\ddagger}$ Corresponding author. Department of Mathematics, Zhejiang University, Hangzhou 310027, China (fangli@zju.edu.cn).
} 
of the basis of $H H^{1}(\mathbf{k} \Gamma)$ is related to the combinatorics, such as Euler's formula, and the topology, such as the genus, of the quiver.

Our second motivation is differential algebra which has its origin in the algebraic study of differential equations [13, 22, 25] and is a natural yet profound extension of commutative algebra and the related algebraic geometry. After many years of developments, the theory has expanded into a vast area in mathematics $[5,13,25]$. Furthermore, differential algebra has found important applications in arithmetic geometry, logic and computational algebra, especially in the profound work of W. Wu on mechanical proof of geometric theorems [27, 28].

Most of the study on differential algebra has been for commutative algebras and fields. Recently, there have been interests to study differential algebra for noncommutative algebras. For instance, in connection with combinatorics, differential structures were found on heap ordered trees [9] and on decorated rooted trees [10].

This paper gives a differential study of the path algebra of a quiver, as a first step in the study of differential structures on Artinian algebras. According to the well-known Gabriel Theorem [2, 3], a basic algebra over an algebraically closed field is a quotient of the path algebra of its Ext-quiver modulo an admissible ideal. More generally, by [18], an Artinian algebra over a perfect field is isomorphic to a quotient of the generalized path algebra of its natural quiver. Thus if we can determine the differential structures on path algebras (resp. generalized path algebras), including their differential ideals, then by taking the quotients of these algebras modulo their differential ideals, we will be able to obtain the differential structure on a basic algebra (resp. an Artinian algebra). For more related references, see [15, 16, 17].

In Section 2 we characterize when a linear operator on a path algebra is a derivation. These characterizations of derivations allow us to obtain in Section 3 a canonical basis of the Lie algebra of derivations on a path algebra and obtain a structure theorem of $\operatorname{Der}(\mathbf{k} \Gamma)$. This structure theorem is then applied to study Lie algebra properties of $\operatorname{Der}(\mathbf{k} \Gamma)$. In Section 4, we focus on three types of derivations of combinatorial nature, namely derivations from the vertices, arrows and faces of the quiver respectively. Dimension formulas of the spaces spanned by these derivations are proved and the relations among them are determined. In Section 5 we give two applications of these dimension formulas. We first revisit Euler's Polyhedron Theorem from a differential viewpoint, and prove that the linear space of edge derivations is the direct sum of the linear spaces of vertex derivations and face derivations. Taking dimensions of the spaces in this direct sum decomposition gives the original formula of Euler. We next apply the combinatorial derivations to obtain a canonical basis for $H H^{1}(\mathbf{k} \Gamma)$. This basis allows us to perform computations in this Lie algebra and to factor it into a semidirect sum of an abelian Lie subalgebra and an Lie ideal. This given basis and the semidirect sum decomposition, i.e. the splitting extension, of the first Hochschild cohomology are in the context of combinatorics and topology. The splitting extension given here is not obtained from a semi-simple Lie algebra by the radical, hence is different from the one in [24] depending upon Levi-Malcev theorem for a finite-dimensional Lie algebra.

2. Derivations on path algebras. The main purpose of this section is to provide necessary and sufficient conditions for a linear operator on a path algebra to be a differential operator.

2.1. Derivations. We briefly recall concepts, notations and facts on differential algebras, Lie algebras and path algebras of quivers. Further details on these three subjects can be found in $[5,10,13]$, in $[12]$ and in $[7,2,3,15]$, respectively. 
Let $\mathbf{k}$ be a field and let $A$ be a $\mathbf{k}$-algebra. Let $\operatorname{Lie}(A)=(A,[]$,$) denote the Lie$ algebra structure on $A$ with the Lie bracket

$$
[x, y]:=x y-y x, \quad x, y \in A
$$

A derivation (or a differential operator) on $A$ is a k-linear map $D: A \rightarrow A$ such that

$$
D(x y)=D(x) y+x D(y), \quad \forall x, y \in A .
$$

Let $\operatorname{Der}(A)$ denote the set of derivations on $A$. Then with the Lie bracket

$$
\left[D_{1}, D_{2}\right]:=D_{1} \circ D_{2}-D_{2} \circ D_{1}, \quad D_{1}, D_{2} \in \operatorname{Der}(A)
$$

$\operatorname{Der}(A)$ is a Lie algebra, called the Lie algebra of derivations on $A$.

For $a \in A$, define the inner derivation

$$
D_{a}: A \rightarrow A, \quad D_{a}(b)=\left(a d_{a}\right)(b):=a b-b a, \quad b \in A .
$$

Then the map

$$
\mathfrak{D}: \operatorname{Lie}(A) \rightarrow \operatorname{Der}(A), \quad \mathfrak{D}(a)=D_{a}, \quad a \in A,
$$

from Eq. (1) is a Lie algebra homomorphism whose kernel is $C(A)$, the center of $A$ and also the zeroth Hochschild cohomology group $H H^{0}(A)$.

The subset $\operatorname{InDer}(A):=\operatorname{im} \mathfrak{D} \subseteq \operatorname{Der}(A)$ is a Lie ideal. The quotient Lie algebra

$$
\operatorname{OutDer}(A):=\operatorname{Der}(A) / \operatorname{InDer}(A)
$$

is called the Lie algebra of outer derivations. As is well-known [21, §11.5], $\operatorname{OutDer}(A)$ is also the first Hochschild cohomology group $H H^{1}(A)$.

We will study $\operatorname{Der}(A)$ and $H H^{1}(A)$ when $A=\mathbf{k} \Gamma$ is the path algebra of a connected quiver $\Gamma$.

2.2. Path algebras. A quiver is a quadruple $\Gamma=(V, E, t, h)$ consisting of a set $V$ of vertices, a set $E$ of arrows and a pair of maps $h, t: E \rightarrow V$ such that for any arrow $a \in E, h(a)$ is called the head of $a$ and $t(a)$ is called the tail of $a$. When there is no danger of confusion, we also denote $\Gamma=(V, E)$. A quiver is called trivial if $E=\emptyset$.

Let $p$ be a path in $\Gamma$ consisting of the ordered list $v_{0}, p_{1}, v_{1}, \cdots, v_{\ell-1}, p_{\ell}, v_{\ell}$, with $v_{i} \in V, 0 \leq i \leq \ell$ and $p_{j} \in E, 1 \leq j \leq \ell$, such that $t\left(p_{j}\right)=v_{j-1}$ and $h\left(p_{j}\right)=v_{j}$, $1 \leq j \leq \ell$. The integer $\ell \geq 0$ is called the length of the path $p$ and is denoted by $\ell(p)$. The path $p$ is expressed as

$$
p=v_{0} p_{1} v_{1} \cdots v_{\ell-1} p_{\ell} v_{\ell}
$$

called the standard decomposition of $p$, and the expression

$$
p=p_{1} \cdots p_{\ell}
$$

is called the decomposition of $p$ into arrows. Both decompositions are unique.

Let $\mathcal{P}$ denote the set of paths of $\Gamma$. For $p \in \mathcal{P}$ let $t(p)$ and $h(p)$ denote the tail and the head of $p$ respectively. 
Let

$$
\mathbf{k} \Gamma=\bigoplus_{p \in \mathcal{P}} \mathbf{k} p
$$

denote the path algebra of $\Gamma$ where the product is given by

$$
p \cdot q:=\delta_{h(p), t(q)} p q:= \begin{cases}p q, & h(p)=t(q) \\ 0, & \text { otherwise }\end{cases}
$$

Here $\delta_{h(p), t(q)}$ is the delta function. To simplify notations, we often suppress the symbol $\cdot$ and denote $p \cdot q=p q$, with the convention that $p q=0$ when $h(p) \neq t(q)$.

We will use the following notations on quivers and their path algebras.

\section{DEFINITION 2.1.}

(a) For two paths $p$ and $q$, denote $p \| q$ and called $p$ and $q$ parallel, if $t(p)=t(q)$ and $h(p)=h(q)$.

(b) For two paths $p$ and $q$, if $q=p r$ (resp. $q=r p$ ) for some path $r$, then call $p$ a tail (resp. head) of $q$ and denote by $p \leq_{t} q$ (resp. $p \leq_{h} q$ ). Such an $r$ is unique for given $p, q$.

(c) A path $p$ is called acyclic if $h(p) \neq t(p)$. The set of acyclic paths is denoted by $\mathcal{P}_{A}$.

(d) A quiver $\Gamma$ is called acyclic if $\Gamma$ has no oriented cycles, that is, if $\mathcal{P} \backslash V=\mathcal{P}_{A}$.

In this paper, we always assume that a quiver $\Gamma$ is finite, that is, its vertex set and arrow set are both finite. By the linearity of a derivation, we have:

Lemma 2.2. Let $A$ be a k-algebra with a linear basis $X$. Then a linear operator $D: A \rightarrow A$ is a derivation if and only if

$$
D(x y)=D(x) y+x D(y), \quad \forall x, y \in X .
$$

In particular, a linear operator $D: \mathbf{k} \Gamma \rightarrow \mathbf{k} \Gamma$ is a derivation if and only if Eq. (4) holds for all $x, y \in \mathcal{P}$.

2.3. Necessary and sufficient conditions for a derivation. We now characterize a derivation on a path algebra $\mathbf{k} \Gamma$ in terms of the paths $\mathcal{P}$ of $\Gamma$. These characterizations will be applied in the next section to determine all derivations on a path algebra.

Let $D: \mathbf{k} \Gamma \rightarrow \mathbf{k} \Gamma$ be a linear operator. Then for any $p \in \mathcal{P}$,

$$
D(p)=\sum_{q \in \mathcal{P}} c_{q}^{p} q
$$

for unique $c_{q}^{p} \in \mathbf{k}$. We will use this notation for the rest of this paper. We also use the convention that, for the empty set $\emptyset$,

$$
\sum_{q \in \emptyset} c_{q}^{p} q=0
$$

TheOrem 2.3. Let $\Gamma$ be a quiver. A linear operator $D: \mathbf{k} \Gamma \rightarrow \mathbf{k} \Gamma$ is a derivation if and only if $D$ satisfies the following conditions. 
(a) For $v \in V$,

$$
D(v)=\sum_{q \in \mathcal{P}_{A}, t(q)=v} c_{q}^{v} q+\sum_{q \in \mathcal{P}_{A}, h(q)=v} c_{q}^{v} q=\sum_{q \in \mathcal{P}_{A}, t(q)=v \text { or } h(q)=v} c_{q}^{v} q .
$$

(b) For $p \in \mathcal{P} \backslash V$,

$$
D(p)=\sum_{q \in \mathcal{P}_{A}, h(q)=t(p)} c_{q}^{t(p)} q p+\sum_{q \| p} c_{q}^{p} q+\sum_{q \in \mathcal{P}_{A}, t(q)=h(p)} c_{q}^{h(p)} p q,
$$

where the coefficients $c_{q}^{p}$ are subject to the following conditions.

(i) For any path $q \in \mathcal{P}_{A}$,

$$
c_{q}^{h(q)}+c_{q}^{t(q)}=0 .
$$

(ii) For any path $p=p_{1} p_{2}$ with $p_{1}, p_{2} \in \mathcal{P} \backslash V$ and $q \| p$, we have

(8)

$$
c_{q}^{p}=c_{q}^{p_{1} p_{2}}= \begin{cases}c_{q_{1}}^{p_{1}}+c_{q_{2}}^{p_{2}}, & \text { if } p_{2} \leq_{h} q \text { with } q=q_{1} p_{2} \text { and } p_{1} \leq_{t} q \text { with } q=p_{1} q_{2}, \\ c_{q_{1}}^{p_{1}}, & \text { if } p_{2} \leq_{h} q \text { with } q=q_{1} p_{2} \text { and } q_{1} \leq_{t} p_{1}, \\ c_{q_{2}}^{p_{2}}, & \text { if } p_{1} \leq_{t} q \text { with } q=p_{1} q_{2} \text { and } q_{2} \leq_{h} p_{2}, \\ 0, & \text { if } p_{2} \leq_{h} q \text { and } p_{1} \leq_{t} q .\end{cases}
$$

Proof. $(\Longrightarrow)$ Let $D: \mathbf{k} \Gamma \rightarrow \mathbf{k} \Gamma$ be a linear operator. For a given $v \in V$, since $v v=v$, we have $D(v)=D(v v)=D(v) v+v D(v)$. Thus

$$
\sum_{q \in \mathcal{P}} c_{q}^{v} q=\left(\sum_{q \in \mathcal{P}} c_{q}^{v} q\right) v+v\left(\sum_{q \in \mathcal{P}} c_{q}^{v} q\right)=\sum_{q \in \mathcal{P}, h(q)=v} c_{q}^{v} q+\sum_{q \in \mathcal{P}, t(q)=v} c_{q}^{v} q
$$

since $q v=0$ unless $h(q)=v$ and $v q=0$ unless $t(q)=v$.

Similarly, from

$$
D(v)=D\left(v^{3}\right)=D(v) v^{2}+v D(v) v+v^{2} D(v)=D(v) v+v D(v) v+v D(v),
$$

we have

$$
\begin{aligned}
\sum_{q \in \mathcal{P}} c_{q}^{v} q & =\left(\sum_{q \in \mathcal{P}} c_{q}^{v} q\right) v+v\left(\sum_{q \in \mathcal{P}} c_{q}^{v} q\right) v+v\left(\sum_{q \in \mathcal{P}} c_{q}^{v} q\right) \\
& =\sum_{q \in \mathcal{P}, h(q)=v} c_{q}^{v} q+\sum_{q \in \mathcal{P}, h(q)=v, t(q)=v} c_{q}^{v} q+\sum_{q \in \mathcal{P}, t(q)=v} c_{q}^{v} q .
\end{aligned}
$$

Comparing this with Eq. (9), we obtain $\sum_{q \in \mathcal{P}, h(q)=v, t(q)=v} c_{q}^{v} q=0$. Then Eq. (5) follows from Eq. (9).

Also, for a given path $p \in \mathcal{P} \backslash V$, we have

$$
\begin{aligned}
D(p) & =D(t(p) p h(p)) \\
& =D(t(p)) p h(p)+t(p) D(p) h(p)+t(p) p D(h(p)) \\
& =D(t(p)) p+t(p) D(p) h(p)+p D(h(p)) \\
& =D(t(p)) p+\sum_{q \| p} c_{q}^{p} q+p D(h(p)) .
\end{aligned}
$$


By Eq. (5), we have

$$
D(t(p)) p=\left(\sum_{q \in \mathcal{P}_{A}, t(q)=t(p)} c_{q}^{t(p)} q+\sum_{q \in \mathcal{P}_{A}, h(q)=t(p)} c_{q}^{t(p)} q\right) p=\sum_{q \in \mathcal{P}_{A}, h(q)=t(p)} c_{q}^{t(p)} q p
$$

since $q p=0$ if $h(q) \neq t(p)$. Similarly, $p D(h(p))=\sum_{q \in \mathcal{P}_{A}, t(q)=h(p)} c_{q}^{h(p)} p q$. This proves Eq. (6).

Thus we only need to prove Eq. (7) and Eq. (8) in order to complete the proof of $(\Longrightarrow)$. For this purpose, we prove a lemma.

\section{LEMMA 2.4 .}

(a) Let $p_{1}, p_{2} \in \mathcal{P}$. Suppose $p_{1} p_{2}=0$ and Eq. (5) and Eq. (8) hold for $p_{1}$ and $p_{2}$. Then $D\left(p_{1} p_{2}\right)=D\left(p_{1}\right) p_{2}+p_{1} D\left(p_{2}\right)$ if and only if, for every $q \in \mathcal{P}$ with $t(q)=p_{1}$ and $h(q)=p_{2}$, Eq. (7) holds.

(b) Let $p_{1}, p_{2} \in \mathcal{P} \backslash V$. Suppose $p_{1} p_{2} \neq 0$ and Eq. (6) holds for $p_{1}, p_{2}$ and $p_{1} p_{2}$. Then $D\left(p_{1} p_{2}\right)=D\left(p_{1}\right) p_{2}+p_{1} D\left(p_{2}\right)$ if and only if, for every $q \| p, E q$. (8) holds.

Proof. (a). Since $p_{1} p_{2}=0$ and $D$ is linear, we have $D\left(p_{1} p_{2}\right)=0$. To compute $D\left(p_{1}\right) p_{2}+p_{1} D\left(p_{2}\right)$, first consider the case when $p_{1}, p_{2}$ are in $V$.

$$
\begin{aligned}
& D\left(p_{1}\right) p_{2}+p_{1} D\left(p_{2}\right) \\
= & \left(\sum_{q \in \mathcal{P}_{A}, h(q)=p_{1}} c_{q}^{p_{1}} q+\sum_{q \in \mathcal{P}_{A}, t(q)=p_{1}} c_{q}^{p_{1}} q\right) p_{2} \\
& \left.+p_{1}\left(\sum_{q \in \mathcal{P}_{A}, h(q)=p_{2}} c_{q}^{p_{2}} q+\sum_{q \in \mathcal{P}_{A}, t(q)=p_{2}} c_{q}^{p_{2}} q\right) \quad \text { (by Eq. (6)) } \sum_{q \in \mathcal{P}_{A}, t(q)=p_{1}, h(q)=p_{2}} c_{q}^{p_{1}} q+c_{q \in \mathcal{P}_{A}, t(q)=p_{1}, h(q)=p_{2}}^{p_{2}} q \quad \quad \text { (since } p_{1} \neq p_{2}\right) \\
= & \sum_{q \in \mathcal{P}_{A}, t(q)=p_{1}, h(q)=p_{2}}\left(c_{q}^{p_{1}}+c_{q}^{p_{2}}\right) q .
\end{aligned}
$$

Thus $D\left(p_{1}\right) p_{2}+p_{1} D\left(p_{2}\right)=0$ if and only if all the coefficients in the last sum are zero. That is, $c_{q}^{h(q)}+c_{q}^{t(q)}=0$ for all $q \in \mathcal{P}$ with $t(q)=p_{1}, h(q)=p_{2}$. This proves (a).

Next consider the case when $p_{1}$ and $p_{2}$ are in $\mathcal{P} \backslash V$. By a similar computation ${ }^{1}$, we have:

$$
D\left(p_{1}\right) p_{2}+p_{1} D\left(p_{2}\right)=\sum_{t(q)=h\left(p_{1}\right), h(q)=t\left(p_{2}\right)}\left(c_{q}^{h\left(p_{1}\right)}+c_{q}^{t\left(p_{2}\right)}\right) p_{1} q p_{2} .
$$

For any two distinct $p_{1}, p_{2} \in \mathcal{P}$ with $t(q)=h\left(p_{1}\right)$ and $h(q)=t\left(p_{2}\right)$, the corresponding $p_{1} q p_{2}$ are non-zero and are distinct paths in the basis $\mathcal{P}$ of $\mathbf{k} \Gamma$. Thus $D\left(p_{1}\right) p_{2}+$ $p_{1} D\left(p_{2}\right)=0$ if and only if all the coefficients in the last sum are zero. That is, $c_{q}^{h\left(p_{1}\right)}+c_{q}^{t\left(p_{2}\right)}=0$ for all $q \in \mathcal{P}$ with $t(q)=h\left(p_{1}\right), h(q)=t\left(p_{2}\right)$. This proves (a) in this case.

\footnotetext{
${ }^{1}$ For details of this and latter computations, see the online version of the paper arXiv:1010.1980v2.
} 
The cases when one of $p_{1}, p_{2}$ is in $V$ and the other one is in $\mathcal{P} \backslash V$ can be verified in the same way.

(b). By Eq. (6) we have

(10) $D\left(p_{1} p_{2}\right)=\sum_{q \in \mathcal{P}_{A}, h(q)=t\left(p_{1}\right)} c_{q}^{t\left(p_{1}\right)} q p_{1} p_{2}+\sum_{q \| p_{1} p_{2}} c_{q}^{p_{1} p_{2}} q+\sum_{q \in \mathcal{A}, t(q)=h\left(p_{2}\right)} c_{p}^{h\left(p_{2}\right)} p_{1} p_{2} q$ since $t\left(p_{1} p_{2}\right)=t\left(p_{1}\right)$ and $h\left(p_{1} p_{2}\right)=h\left(p_{2}\right)$. Similarly,

$$
D\left(p_{1}\right) p_{2}=\left(\sum_{q \in \mathcal{P}_{A}, h(q)=t\left(p_{1}\right)} c_{q}^{t\left(p_{1}\right)} q p_{1}+\sum_{q_{1} \| p_{1}} c_{q_{1}}^{p_{1}} q_{1}+\sum_{q \in \mathcal{P}_{A}, t(q)=h\left(p_{1}\right)} c_{q}^{h\left(p_{1}\right)} p_{1} q\right) p_{2} .
$$

Since $h(q) \neq t(q)=h\left(p_{1}\right)=t\left(p_{2}\right)$, we have $q p_{2}=0$ for $q$ in the last sum. Thus we obtain

$$
D\left(p_{1}\right) p_{2}=\sum_{q \in \mathcal{P}_{A}, h(q)=t\left(p_{1}\right)} c_{q}^{t\left(p_{1}\right)} q p_{1} p_{2}+\sum_{q_{1} \| p_{1}} c_{q_{1}}^{p_{1}} q_{1} p_{2}
$$

By the same argument, we have

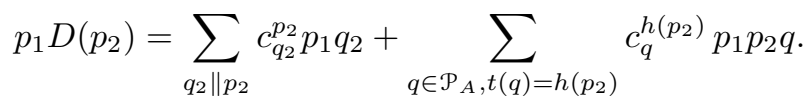

Thus by equations (10), (11) and (12), we see that $D\left(p_{1} p_{2}\right)=D\left(p_{1}\right) p_{2}+p_{1} D\left(p_{2}\right)$ if and only if

$$
\sum_{q \| p_{1} p_{2}} c_{q}^{p_{1} p_{2}} q=\sum_{q_{1} \| p_{1}} c_{q_{1}}^{p_{1}} q_{1} p_{2}+\sum_{q_{2} \| p_{2}} c_{q_{2}}^{p_{2}} p_{1} q_{2}
$$

In the sum on the left hand side, the paths $q \| p_{1} p_{2}$ can be divided into the disjoint union of the following four subsets:

$$
\begin{aligned}
& \mathcal{P}_{1}:=\left\{q \in \mathcal{P} \mid p \| p_{1} p_{2}, p_{1} \leq_{t} q \text { and } p_{2} \leq_{h} q\right\}, \\
& \mathcal{P}_{2}:=\left\{q \in \mathcal{P} \mid q \| p_{1} p_{2}, p_{1} \leq_{t} q \text { and } p_{2} \not_{h} q\right\}, \\
& \mathcal{P}_{3}:=\left\{q \in \mathcal{P} \mid q \| p_{1} p_{2}, p_{1} \not_{t} q \text { and } p_{2} \leq_{h} q\right\}, \\
& \mathcal{P}_{4}:=\left\{q \in \mathcal{P} \mid q \| p_{1} p_{2}, p_{1} \not_{t} q \text { and } p_{2} \not_{h} q\right\} .
\end{aligned}
$$

Thus the left hand side of Eq. (13) becomes

$$
\sum_{q \| p_{1} p_{2}} c_{q}^{p_{1} p_{2}} q=\sum_{q \in \mathcal{P}_{1}} c_{q}^{p_{1} p_{2}} q+\sum_{q \in \mathcal{P}_{2}} c_{q}^{p_{1} p_{2}} q+\sum_{q \in \mathcal{P}_{3}} c_{q}^{p_{1} p_{2}} q+\sum_{q \in \mathcal{P}_{4}} c_{q}^{p_{1} p_{2}} q .
$$

By the definitions of $\leq_{t}$ and $\leq_{h}$, for the two sums on the right hand side of Eq. (13), we have respectively

$$
\left\{q_{1} p_{2} \mid q_{1} \| p_{1}\right\}=\mathcal{P}_{1} \cup \mathcal{P}_{3}
$$

and

$$
\left\{p_{1} q_{2} \mid q_{2} \| p_{2}\right\}=\mathcal{P}_{1} \cup \mathcal{P}_{2} .
$$


Thus the right hand side of Eq. (13) becomes (20)

$$
\sum_{q \in \mathcal{P}_{1} \text { with } q=q_{1} p_{2}=p_{1} q_{2}}\left(c_{q_{1}}^{p_{1}}+c_{q_{2}}^{p_{2}}\right) q+\sum_{q \in \mathcal{P}_{3} \text { with } q=q_{1} p_{2}} c_{q_{1}}^{p_{1}} q_{1} p_{2}+\sum_{q \in \mathcal{P}_{2}} \text { with } c_{q=p_{1} q_{2}} c_{q_{2}}^{p_{2}} p_{1} q_{2} .
$$

Now comparing the coefficients on the two sides of Eq. (13) using Eq. (18) and Eq. (20), we obtain Eq. (8).

Now we return to the proof of Theorem 2.3. For any path $p \in \mathcal{P} \backslash V$ with $t(p) \neq$ $h(p)$, we have $t(p) h(p)=0$. So applying Lemma 2.4.(a) to $p_{1}=t(p)$ and $p_{2}=h(p)$ in Eq. (5), we obtain Eq. (7).

Finally let $p=p_{1} p_{2}$ with $p_{1}, p_{2} \in \mathcal{P} \backslash V$. Applying Lemma 2.4.(b) to $p=p_{1} p_{2}$, we obtain Eq. (8).

$(\Longleftarrow)$ Suppose a linear operator $D: \mathbf{k} \Gamma \rightarrow \mathbf{k} \Gamma$ is given by Eq. (5) and (6) subject to the conditions Eq. (7) and (8). By Lemma 2.2, to show that $D$ is a derivation we just need to show that Eq. (4) holds for $X=\mathcal{P}$. Thus we only need to verify Eq. (4) in the following four cases.
(a) $x, y \in V$;
(b) $x \in V, y \in \mathcal{P} \backslash V$;
(c) $x \in \mathcal{P} \backslash V, y \in V$;
(d) $x, y \in \mathcal{P} \backslash V$.

The checking of each case is routine and is omitted.

2.4. A variation of Theorem 2.3. For the convenience of later applications, we give another formulation of Theorem 2.3 on the condition of a derivation on a path algebra.

Corollary 2.5. Let $\Gamma$ be a quiver. A linear operator $D: \mathbf{k} \Gamma \rightarrow \mathbf{k} \Gamma$ is a derivation if and only if $D$ is determined by its action on the basis $\mathcal{P}$ as follows.

(a) Let $v \in V$. Then

$$
D(v)=\sum_{q \in \mathcal{P}_{A}, t(q)=v} c_{q}^{t(q)} q-\sum_{q \in \mathcal{P}_{A}, h(q)=v} c_{q}^{t(q)} q .
$$

(b) Let $p \in \mathcal{P} \backslash V$. Then

$$
D(p)=\sum_{q \in \mathcal{P}_{A}, t(q)=h(p)} c_{q}^{t(q)} p q+\sum_{q \| p} c_{q}^{p} q-\sum_{q \in \mathcal{P}_{A}, h(q)=t(p)} c_{q}^{t(q)} q p,
$$

where the coefficients $c_{q}^{p}$ are subject to the following condition: For any path $p=p_{1} p_{2}$ with $p_{1}, p_{2} \in \mathcal{P} \backslash V$ and $q \| p$, we have

$$
c_{q}^{p}=c_{q}^{p_{1} p_{2}}= \begin{cases}c_{q_{1}}^{p_{1}}+c_{q_{2}}^{p_{2}}, & \text { if } p_{2} \leq_{h} q \text { with } q=q_{1} p_{2} \text { and } p_{1} \leq_{t} q \text { with } q=p_{1} q_{2}, \\ c_{q_{1}}^{p_{1}}, & \text { if } p_{2} \leq_{h} q \text { with } q=q_{1} p_{2} \text { and } q_{1} \leq_{t} p_{1}, \\ c_{q_{2}}^{p_{2}}, & \text { if } p_{1} \leq_{t} q \text { with } q=p_{1} q_{2} \text { and } q_{2} \leq_{h} p_{2}, \\ 0, & \text { if } p_{2} \underline{\leq}_{h} q \text { and } p_{1} \not_{t} q .\end{cases}
$$

Proof. We only need to show that the condition Eq (7) imposed to Eq. (5) and Eq. (6) in Theorem 2.3 amount to Eq. (21) and Eq. (22). First, applying Eq. (7), that is $c_{p}^{h(p)}=-c_{p}^{t(p)}$ for $p \in \mathcal{P} \backslash V$, to Eq. (5) gives us Eq. (21). 
Similarly apply Eq. (7) to Eq. (6). For the coefficients in the first sum, we have $c_{q}^{h(p)}=c_{q}^{t(q)}$ by the restriction of the sum. For the coefficients in the third sum of Eq. (6) we have $c_{q}^{t(p)}=c_{p}^{h(q)}=-c_{q}^{t(q)}$. Thus the first and third sums in Eq. (6) agree with the corresponding sums in Eq. (22). This is what we need.

3. The Structure of Lie algebra $\operatorname{Der}(\mathbf{k} \Gamma)$ for a quiver $\Gamma$. In this section, we apply the characterizations (Theorem 2.3 and Corollary 2.5) of a derivation on a path algebra to study the Lie algebra $\operatorname{Der}(\mathbf{k} \Gamma)$. We first display a canonical basis for this Lie algebra and then use the basis to establish the multiplication structure of this Lie algebra. As applications, basic properties of this Lie algebra are studies.

3.1. The derivation $D_{r, s}$. Let $r \in E$ and $s \| r$. We construct a linear operator

$$
D_{r, s}: \mathbf{k} \Gamma \rightarrow \mathbf{k} \Gamma
$$

by defining $D_{r, s}(p)$ for $p \in \mathcal{P}$ by induction on the length $\ell(p)$ of $p$.

When $\ell(p)=0$, i.e., $p \in V$, we define

$$
D_{r, s}(p)=0 .
$$

Assume that $D_{r, s}(p)$ have been defined for $p \in \mathcal{P}$ with $\ell(p)=n \geq 0$. Consider $p \in \mathcal{P}$ with $\ell(p)=n+1$. Then $p=p_{1} \tilde{p}$ with $p_{1} \in E$ and $\tilde{p} \in \mathcal{P}$ with $\ell(\tilde{p})=n$. We then define

$$
D_{r, s}(p)= \begin{cases}s \tilde{p}+p_{1} D_{r, s}(\tilde{p}), & p_{1}=r \\ p_{1} D_{r, s}(\tilde{p}), & p_{1} \neq r\end{cases}
$$

Proposition 3.1. For a quiver $\Gamma=(V, E)$, let $r \in E$ and $s \| r$. The linear operator $D_{r, s}$ recursively defined by Eqs. (23) and (24) have the following explicit formula. For any $p \in \mathcal{P}$ with the standard decomposition $p=v_{0} p_{1} v_{1} \cdots p_{k} v_{k}$ with $v_{0}, \cdots, v_{k} \in V, p_{1}, \cdots, p_{k} \in E$, we have

$$
D_{r, s}(p)= \begin{cases}0, & k=0 \\ \sum_{i=1}^{k} v_{0} p_{i, 1} v_{1} \cdots p_{i, k} v_{k}, & k>0\end{cases}
$$

where $p_{i, j}$ or, more precisely, $p_{i, j}^{(r, s)}$ is defined by

$$
p_{i, j}:=p_{i, j}^{(r, s)}:= \begin{cases}s, & i=j, p_{i}=r, \\ 0, & i=j, p_{i} \neq r, \\ p_{j}, & i \neq j .\end{cases}
$$

For example, for $p \in \mathcal{P}$ with standard decomposition $p=v_{0} p_{1} v_{1} p_{2} v_{2} p_{3} v_{3} p_{4} v_{4}$ where $p_{1}, p_{3}=r$ and $p_{2}, p_{4} \neq r$, we have

$$
D_{r, s}(p)=v_{0} s v_{1} p_{2} v_{2} r v_{3} p_{4} v_{4}+v_{0} r v_{1} p_{2} v_{2} s v_{3} p_{4} v_{4} .
$$

Proof. Let $D$ be defined by Eq. (25). We just need to show that $D_{r, s}(p)=D(p)$ for all $p \in \mathcal{P}$. We prove this by induction on $\ell(p)$. When $\ell(p)=0$, then $D_{r, s}(p)=$ $0=D(p)$ by the definitions of $D_{r, s}$ and $D$. Assume the equation holds for $\ell(p)=k$ for $k \geq 0$, and consider $p \in \mathcal{P}$ with $\ell(p)=k+1$. Then $p=v_{0} p_{1} \tilde{p}=p_{1} \tilde{p}$ with $p_{1} \in E$ 
and $\tilde{p} \in \mathcal{P}$ with $\ell(\tilde{p})=k$. Let $\tilde{p}=v_{1} p_{2} v_{2} \cdots p_{k+1} v_{k+1}$ be the standard decomposition of $\tilde{p}$. Then by the induction hypothesis we have

$$
\begin{aligned}
D_{r, s}(p) & = \begin{cases}s \tilde{p}+p_{1} D_{r, s}(\tilde{p}), & p_{1}=r \\
p_{1} D_{r, s}(\tilde{p}), & p_{1} \neq r\end{cases} \\
& = \begin{cases}v_{0} s v_{1} p_{2} v_{2} \cdots p_{k+1} v_{k+1}+v_{0} p_{1} \sum_{i=2}^{k+1} v_{1} p_{i, 2} v_{2} \cdots p_{i, k+1} v_{k+1}, & p_{1}=r \\
v_{0} p_{1} \sum_{i=2}^{k+1} v_{1} p_{i, 2} v_{2} \cdots p_{i, k+1} v_{k+1}, & p_{1} \neq r\end{cases} \\
& = \begin{cases}v_{0} s v_{1} p_{2} v_{2} \cdots p_{k+1} v_{k+1}+\sum_{i=2}^{k+1} v_{0} p_{1} v_{1} p_{i, 2} v_{2} \cdots p_{i, k+1} v_{k+1}, & p_{1}=r \\
\sum_{i=2}^{k+1} v_{0} p_{1} v_{1} p_{i, 2} v_{2} \cdots p_{i, k+1} v_{k+1}, & p_{1} \neq r\end{cases} \\
& =\sum_{i=1}^{k+1} v_{0} p_{i, 1} v_{1} \cdots p_{i, k+1} v_{k+1},
\end{aligned}
$$

where $p_{i, j}$ is defined by Eq. (26). Since this agrees with $D(p)$, the induction is completed.

Theorem 3.2. For $r \in E$ and $s \| r$, the linear operator $D_{r, s}: \mathrm{k} \Gamma \rightarrow \mathrm{k} \Gamma$ defined by Eq. (23) and Eq. (24) is a derivation.

Proof. By Lemma 2.2, we only need to verify

$$
D_{r, s}(p q)=D_{r, s}(p) q+p D_{r, s}(q), \quad \forall p, q \in \mathcal{P} .
$$

We will prove this by induction on $\ell(p)$.

Let $\ell(p)=0$. Then $p q=q$ if $p=t(q)$ and $p q=0$ if $p \neq t(q)$. First consider the case when $D_{r, s}(q)=0$. Then both sides of Eq. (27) are zero. So we are done. Next consider the case when $D_{r, s}(q) \neq 0$. Then we have $t\left(D_{r, s}(q)\right)=t(q)$ by the definition of $D_{r, s}(q)$. Thus if $t(q) \neq p$, then both sides of Eq. (27) are zero and we are done again. If $t(q)=p$, then both sides of Eq. (27) equal to $D_{r, s}(q)$, as needed.

Next assume that Eq. (27) has been proved for $p \in \mathcal{P}$ with $\ell(p)=n \geq 0$ and consider $p \in \mathcal{P}$ with $\ell(p)=n+1$. Then we can write $p=p_{1} \tilde{p}$ and obtain

$$
\begin{aligned}
D_{r, s}(p q) & =D_{r, s}\left(p_{1} \tilde{p} q\right) \\
& =\left\{\begin{array}{ll}
s \tilde{p} q+p_{1} D_{r, s}(\tilde{p} q), & p_{1}=r, \\
p_{1} D_{r, s}(\tilde{p} q), & p_{1} \neq r .
\end{array} \quad\right. \text { by Eq. (24)) } \\
& =\left\{\begin{array}{ll}
s \tilde{p} q+p_{1}\left(D_{r, s}(\tilde{p}) q+\tilde{p} D_{r, s}(q)\right), & p_{1}=r, \\
p_{1}\left(D_{r, s}(\tilde{p}) q+\tilde{p} D_{r, s}(q)\right), & p_{1} \neq r .
\end{array} \quad\right. \text { (by induction hypothesis) } \\
& =\left\{\begin{array}{ll}
D_{r, s}(p) q+p D_{r, s}(q), & p_{1}=r, \\
D_{r, s}(p) q+p D_{r, s}(q), & p_{1} \neq r .
\end{array} \quad\right. \text { (by Eq. (24)) }
\end{aligned}
$$

This completes the induction.

As an immediate consequence of Theorem 2.3 and Theorem 3.2, we prove the following existence theorem of derivations on path algebras. Note that the zero map on any algebra is a derivation.

COROLlary 3.3. There is a nonzero derivation on the path algebra $\mathrm{k} \Gamma$ of a quiver $\Gamma$ if and only if $\Gamma$ is a non-trivial quiver, that is, $\Gamma$ has at least one arrow. Equivalently, $\operatorname{Der}(\mathbf{k} \Gamma)$ is a non-zero Lie algebra if and only if $\Gamma$ is a non-trivial quiver. 
Proof. Suppose $\Gamma$ contains only vertices. Let $D: \mathrm{k} \Gamma \rightarrow \mathrm{k} \Gamma$ be a derivation. Then by Eq. (5), we have $D(v)=\sum_{q \in \mathcal{P}_{A}, t(q)=v \text { or } h(q)=v} c_{q}^{v} q$. Since $\mathcal{P}_{A}=\emptyset$ in this case, $D(v)=0$ for all $v \in V$. Since $V$ is a basis of $\mathbf{k} \Gamma, D$ is the zero map.

Conversely, suppose $\Gamma$ contains an arrow $p_{0}$. Then we have the derivation $D_{p_{0}, p_{0}}$ by Theorem 3.2. Since $D_{p_{0}, p_{0}}\left(p_{0}\right)=p_{0}$ is nonzero, we have obtained a non-zero derivation on $\mathrm{k} \Gamma$.

3.2. A canonical basis of $\operatorname{Der}(\mathbf{k} \Gamma)$. We now display a canonical basis of the Lie algebra $\operatorname{Der}(\mathbf{k} \Gamma)$ for a quiver $\Gamma$. For a given $s \in \mathcal{P}$, we have the inner derivation

$$
D_{s}: \mathbf{k} \Gamma \rightarrow \mathbf{k} \Gamma, \quad D_{s}(q)=s q-q s, \quad \forall q \in \mathcal{P} .
$$

TheOREM 3.4. Let $\Gamma$ be a quiver. A basis of the Lie algebra $\operatorname{Der}(\mathbf{k} \Gamma)$ is given by the set

$$
\mathfrak{B}:=\mathfrak{B}_{1} \cup \mathfrak{B}_{2}
$$

where

$$
\mathfrak{B}_{1}:=\left\{D_{s} \mid s \in \mathcal{P}_{A}\right\} \quad \text { and } \quad \mathfrak{B}_{2}:=\left\{D_{r, s} \mid r \in E, s \| r\right\} .
$$

Thus $\operatorname{Der}(\mathbf{k} \Gamma)=\mathfrak{D}_{1} \oplus \mathfrak{D}_{2}$ where $\mathfrak{D}_{i}$ are the $\mathbf{k}$-linear space with bases $\mathfrak{B}_{i}$ for $i=1,2$.

We will call $\mathfrak{B}$ the canonical basis of $\operatorname{Der}(\mathbf{k} \Gamma)$.

Proof. Since the operators in $\mathfrak{B}$ are derivations by Theorem 3.2 , we only need to verify that the operators in $\mathfrak{B}$ are linearly independent and that $\mathfrak{B}$ spans the whole space of derivations.

Step 1. $\mathfrak{B}$ is linearly independent. Suppose there are $c_{s}, c_{r, s} \in \mathbf{k}$ such that

$$
D:=\sum_{s \in \mathcal{P}_{A}} c_{s} D_{s}+\sum_{r \in E, s \| r} c_{r, s} D_{r, s}=0 .
$$

Then for any given $s_{0} \in \mathcal{P}_{A}$, by the definitions of $D_{s}$ and $D_{r, s}$ we have

$$
\begin{aligned}
& 0=D\left(h\left(s_{0}\right)\right) \\
&=\sum_{s \in \mathcal{P}_{A}} c_{s} D_{s}\left(h\left(s_{0}\right)\right) \\
&=\sum_{s \in \mathcal{P}_{A}} c_{s}\left(s h\left(s_{0}\right)-h\left(s_{0}\right) s\right) \\
&=\sum_{s \in \mathcal{P}_{A}}, h(s)=h\left(s_{0}\right) \\
& c_{s} s-\sum_{s \in \mathcal{P}_{A}, t(s)=h\left(s_{0}\right)} c_{s} s .
\end{aligned}
$$

Since $h(s) \neq t(s)$ in the sums, the index sets of the two sums are disjoint. Thus both of the sums equal to zero and hence $c_{s}=0$ for all $s \in \mathcal{P}_{A}$ with $h(s)=h\left(s_{0}\right)$. In particular, $c_{s_{0}}=0$. Thus,

$$
D=\sum_{r \in E, s \| r} c_{r, s} D_{r, s} .
$$

Further, for any given $r_{0} \in E$ and $s_{0} \| r_{0}$, by the definitions of $D_{s}$ and $D_{r, s}$ we have $0=D\left(r_{0}\right)=\sum_{s_{0} \neq s \in \mathcal{P}, s \| r_{0}} c_{r_{0}, s} s+c_{r_{0}, s_{0}} s_{0}$, and hence $c_{r_{0}, s_{0}}=0$. 
Thus we have proved that $\mathfrak{B}$ is linearly independent.

Step 2. $\mathfrak{B}$ is a spanning set of derivations on $\mathbf{k} \Gamma$. Let $D: \mathbf{k} \Gamma \rightarrow \mathbf{k} \Gamma$ be a given derivation. Then $D$ is defined by Eq. (21) and Eq. (22) in Corollary 2.5. In particular, for $p \in E$, by Eq. (22) we have

$$
D(p)=\sum_{q \in \mathcal{P}_{A}, t(q)=h(p)} c_{q}^{t(q)} p q+\sum_{q \| p} c_{q}^{p} q-\sum_{q \in \mathcal{P}_{A}, h(q)=t(p)} c_{q}^{t(q)} q p
$$

for certain coefficients $c_{q}^{t(q)} \in \mathbf{k}$ where $q \in \mathcal{P}_{A}$ with $t(q)=h(p)$ and $c_{q}^{p} \in \mathbf{k}$ where $q \| p$.

We claim that $D$ agrees with the operator $\bar{D}$ defined by the linear combination

$$
\bar{D}=-\sum_{s \in \mathcal{P}_{A}} c_{s}^{t(s)} D_{s}+\sum_{r \in E, s \in \mathcal{P}, h(s)=h(r), t(s)=t(r)} c_{s}^{r} D_{r, s},
$$

obtained by the same coefficients appeared in Eq. (29). As a linear combination of derivations, $\bar{D}$ is also a derivation. Any path in $\mathcal{P}$ is either a vertex or a product of arrows. Thus by the product rule of derivations, to show the equality of $D$ and $\bar{D}$, we only need to verify that $D(q)=\bar{D}(q)$ for each $q=v \in V$ and $q=p \in E$.

First let $q=v \in V$. Since $D_{r, s}(v)=0$, we have

$\bar{D}(v)=-\sum_{s \in \mathcal{P}_{A}} c_{s}^{t(s)} D_{s}(v)=-\sum_{s \in \mathcal{P}_{A}} c_{s}^{t(s)}(s v-v s)=-\sum_{s \in \mathcal{P}_{A}, h(s)=v} c_{s}^{t(s)} s v+\sum_{s \in \mathcal{P}_{A}, t(s)=v} c_{s}^{t(s)} v s$.

Here the last equality follows since, in the first sum, $s v$ is $s$ if $h(s)=v$ and is zero otherwise, and in the second sum, $v s$ is $s$ if $t(s)=v$ and is zero otherwise. Thus $\bar{D}(v)$ agrees with $D(v)$ as defined in Eq. (21).

Next let $q=p \in E$. Then $D_{r, s}(p)$ is $s$ if $r=p$ and is 0 otherwise. Thus we have

$$
\begin{aligned}
\bar{D}(p) & =-\sum_{s \in \mathcal{P}_{A}} c_{s}^{t(s)} D_{s}(p)+\sum_{r \in E, s \| r} c_{s}^{r} D_{r, s}(p) \\
& =-\sum_{s \in \mathcal{P}_{A}} c_{s}^{t(s)}(-p s+s p)+\sum_{s \| p} c_{s}^{p} s \\
& =\sum_{s \in \mathcal{P}_{A}, t(s)=h(p)} c_{s}^{t(s)} p s-\sum_{s \in \mathcal{P}_{A}, h(s)=t(p)} c_{s}^{t(s)} s p+\sum_{s \| p} c_{s}^{p} s .
\end{aligned}
$$

This agrees with $D(p)$ in Eq. (29). Thus $\bar{D}=D$, showing that $\mathfrak{B}$ spans $\operatorname{Der}(\mathbf{k} \Gamma)$.

The proof of Theorem 3.4 is completed.

3.3. A Structure theorem of $\operatorname{Der}(\mathbf{k} \Gamma)$. Let $p \in E$ and let $\sum_{i=1}^{k} c_{i} q_{i} \in \mathbf{k} \Gamma$ where $c_{i} \in \mathbf{k}$ and $q_{i} \in \mathcal{P}$ with $q_{i} \| p$. We denote

$$
D_{p, \sum_{i=1}^{k} c_{i} q_{i}}=\sum_{i=1, q_{i} \| p}^{k} c_{i} D_{p, q_{i}}
$$

Theorem 3.5. (Basis Theorem of $\operatorname{Der}(\mathbf{k} \Gamma)$ ) For derivations in $\operatorname{Der}(\mathbf{k} \Gamma)$, the following relations hold.

$$
\begin{aligned}
{\left[D_{p}, D_{r}\right] } & =D_{[p, r]}, \quad \text { for } \quad p, r \in \mathcal{P}, \\
{\left[D_{p}, D_{r, s}\right] } & =-D_{D_{r, s}(p)}, \quad \text { for } \quad r \in E, p, s \in \mathcal{P}, s \| r \\
{\left[D_{r, s}, D_{p, q}\right] } & =D_{p, D_{r, s}(q)}-D_{r, D_{p, q}(s)}, \quad \text { for } \quad r, p \in E, s\|r, q\| p .
\end{aligned}
$$


Proof. Eq. (31) follows from the fact that the map in Eq. (2) is a Lie algebra homomorphism.

Eq. (32) follows from a direct computation using the definitions of $D_{p}$ and $D_{r, s}$.

Finally let $r, p \in E$ and $s \| r$ and $q \| p$. Since both sides of Eq. (33) are derivations, by the product rule of derivations, we only need to prove that, for $t \in E$, the following holds

$$
\left[D_{r, s}, D_{p, q}\right](t)=D_{p, D_{r, s}(q)}(t)-D_{r, D_{p, q}(s)}(t) .
$$

Let such a $t$ be given. If $t \neq r, p$, then both sides of Eq. (34) are zero. If $t=p$, then both sides of Eq. (34) equal to $D_{r, s}(q)-D_{p, q}(s)$ if $t=r$ and equal to $D_{r, s}(q)$ if $t \neq r$. If $t=r$, then both sides of Eq. (34) equal to $D_{r, s}(q)-D_{p, q}(s)$ if $t=p$ and equal to $-D_{p, q}(s)$ if $t \neq p$. This proves Eq. (33).

Note that we usually do not require $D_{p}$ or $D_{r}$ to be in $\mathfrak{B}_{1}$. So $h(p)=t(p)$ or $h(r)=t(r)$ are allowed. In fact, even when $D_{p}$ and $D_{r}$ are in $\mathfrak{B}_{1}, D_{[r, p]}=D_{r p}-D_{p r}$ might not be in the linear space $\mathfrak{D}_{1}$ spanned by $\mathfrak{B}_{1}$. For example, if $r$ is a path from a vertex $v_{1}$ to another vertex $v_{2} \neq v_{1}$ and $p$ is a path from $v_{2}$ to $v_{1}$, then $D_{r}$ and $D_{p}$ are in $\mathfrak{B}_{1}$. But $p r$ and $r p$ are both oriented cycles, so $D_{p r}$ and $D_{r p}$ are not in $\mathfrak{B}_{1}$.

3.4. Ideals and nilpotency. We next apply Theorem 3.5 to study Lie algebra properties of $\operatorname{Der}(\mathbf{k} \Gamma)$.

Proposition 3.6. For any non-trivial quiver $\Gamma$, the Lie algebra $\operatorname{Der}(\mathbf{k} \Gamma)$ is not nilpotent.

Proof. By the well-known Engel theorem [12], a Lie algebra $\mathfrak{g}$ is nilpotent if and only if for all its elements $g$, the adjoint derivation

$$
\operatorname{ad} g: \mathfrak{g} \rightarrow \mathfrak{g}, \quad h \mapsto[g, h], \quad h \in \mathfrak{g},
$$

is nilpotent. Let $p$ be an arrow in $\Gamma$. Then we have $\operatorname{ad} D_{p, p}\left(D_{p}\right)=D_{p}$ and thus for any natural number $n,\left(\boldsymbol{a d} D_{p, p}\right)^{n}\left(D_{p}\right)=D_{p}$. So $\mathbf{a d} D_{p, p}$ is not nilpotent.

TheOREM 3.7. Let $\Gamma$ be an acyclic quiver. Let $\mathfrak{B}_{1}, \mathfrak{B}_{2} \subseteq \mathfrak{B}$ and $\mathfrak{D}_{1}=\mathbf{k} \mathfrak{B}_{1}$, $\mathfrak{D}_{2}=\mathbf{k} \mathfrak{B}_{2}$ be defined in Theorem 3.4. Then,

(a) $\mathfrak{D}_{1}$ (resp. $\mathfrak{D}_{2}$ ) is an ideal (resp. subalgebra) of the Lie algebra $\operatorname{Der}(\mathbf{k} \Gamma)$;

(b) (Structure theorem of $\operatorname{Der}(\mathbf{k} \Gamma)$ ) $\operatorname{Der}(\mathbf{k} \Gamma)$ is a semi-direct sum of the Lie ideal $\mathfrak{D}_{1}$ and the Lie subalgebra $\mathfrak{D}_{2}$, that is,

$$
\operatorname{Der}(\mathbf{k} \Gamma)=\mathfrak{D}_{1} \ltimes \mathfrak{D}_{2} ;
$$

(c) If $\Gamma$ is also a finite quiver, then $\mathfrak{D}_{1}$ is a nilpotent Lie algebra.

Proof. (a). Since $\Gamma$ does not contain any oriented cycles, for any $p, r \in \mathcal{P}_{A}$, we have $r p \in \mathcal{P}_{A}$ unless $r p=0$ and $p r \in \mathcal{P}_{A}$ unless $p r=0$. Thus by Eq. (31), $\mathfrak{D}_{1}$ is closed under the Lie bracket. Further, for $p \in \mathcal{P}_{A}$ and $r \in E, s \| r$, by the acyclicity of $r$ and the definition of $D_{r, s}$ in Eq. $(24), D_{r, s}(p)=\sum_{i} c_{i} q_{i}$ for $q_{i} \in \mathcal{P} \backslash V$. Since $\mathcal{P} \backslash V=\mathcal{P}_{A}$ by assumption, we see that $D_{D_{r, s}(p)}=\sum_{i} c_{i} D_{q_{i}}$ is in $\mathfrak{D}_{1}$. Thus $\left[D_{r, s}, D_{p}\right]$ is in $\mathfrak{D}_{1}$ by Eq. (32). Since $\operatorname{Der}(\mathbf{k} \Gamma)=\mathfrak{D}_{1} \oplus \mathfrak{D}_{2}$ by Theorem 3.4, this proves that $\mathfrak{D}_{1}$ is an ideal of $\operatorname{Der}(\mathbf{k} \Gamma)$. 
By Eq. (33), $\mathfrak{D}_{2}$ is a Lie subalgebra of $\operatorname{Der}(\mathbf{k} \Gamma)$.

(b). This follows from Item (a) and Theorem 3.4.

(c). We first note that the minimal length of $\mathcal{P}^{\prime}:=\mathcal{P} \backslash V$ is one. We then note that, for

$$
\mathcal{P}^{(2)}:=\left[\mathcal{P}^{\prime}, \mathcal{P}^{\prime}\right]:=\left\{[p, q]=p q-q p \mid p, q \in \mathcal{P}^{\prime}\right\},
$$

the minimal length is two unless $\left[\mathcal{P}^{\prime}, \mathcal{P}^{\prime}\right]=0$. Let $\mathfrak{g}:=\mathbf{k}(\mathcal{P} \backslash V)$. Since $\mathcal{P}^{(2)} \subseteq \mathfrak{g}, \mathfrak{g}$ is a Lie subalgebra of $\mathbf{k} \Gamma$. By an inductive argument, we see that, for the recursively defined $\mathcal{P}^{(n+1)}:=\left[\mathcal{P}^{\prime}, \mathcal{P}^{(n)}\right]$, its minimal length is $n+1$ unless $\mathcal{P}^{(n+1)}=0$.

On the other hand, by our acyclicity and finiteness assumptions on $\Gamma$, the lengths of paths in $\Gamma$ is bounded by $|V|$ : suppose there is a path $p$ of length $|V|+1$ with its standard decomposition $p=v_{0} p_{1} v_{1} \cdots v_{|V|} p_{|V|+1} v_{|V|+1}$. Then there are $0 \leq i<j \leq$ $|V|+1$ such that $v_{i}=v_{j}$. This shows that $p$ contains an oriented cycle, contradicting the acyclicity assumption.

Combining the above two points, we see that $\mathcal{P}^{(n)}=0$ for large enough $n$. Thus $\mathfrak{g}$ is nilpotent.

Under the acyclic assumption, we have $\mathcal{P} \backslash V=\mathcal{P}_{A}$. So the Lie algebra homomorphism $\mathcal{D}: \mathbf{k} \Gamma \rightarrow \operatorname{Der}(\mathbf{k} \Gamma)$ from Eq. (2) sends the above Lie algebra $\mathfrak{g}$ to $\mathfrak{D}_{A}=\mathbf{k} \mathfrak{B}_{1}$ surjectively. Thus $\mathfrak{D}_{A}$ is nilpotent.

We note that when the restriction that $\Gamma$ is acyclic is removed, the first statement of Theorem 3.7 is no longer true. This is because in Eq. (31): $\left[D_{p}, D_{r}\right]=D_{[p, r]}$, the right hand side might not be in $\mathfrak{B}_{1}$ even if $D_{p}$ and $D_{r}$ are. See the remark and example after Theorem 3.5.

3.5. Inner derivations and the canonical basis. We now express inner derivations in $\mathrm{InDerk} \Gamma$ in terms of the canonical basis.

Proposition 3.8. Let $q \in \mathcal{P}$ be such that $h(q)=t(q)$. Let $v_{0}=h(q)$. We have

$$
D_{q}=\sum_{p \in E, t(p)=v_{0}} D_{p, q p}-\sum_{r \in E, h(r)=v_{0}} D_{r, r q} .
$$

Proof. Note that both sides of the equation are derivations and $\mathrm{k} \Gamma$ is generated by $V \cup E$ as a k-algebra. So by the product formula of derivations, we only need to verify that the two sides agree when acting on $V$ and $E$. This follows from a routine computation.

4. Combinatorial derivations and their relations. In this section, we study combinatorial derivations on a path algebra $\mathrm{k} \Gamma$, namely derivations from the combinatorial objects of vertices, edges and faces of $\Gamma$. We define various relation matrices, study their ranks and obtain dimensional formulas of these derivations. These dimensional formula will be applied in the next section to give a strengthened form of Euler's polyhedron formula and to determine the structure of the Lie algebra $H H^{1}(\mathrm{k} \Gamma)$ in the context of combinatorics and topology.

4.1. Combinatorial derivations and their relation matrices. We will consider a quiver $\Gamma$ of genus $g$ which is defined to be a quiver together with a fixed embedding of $\Gamma$ into an oriented surface $S$ of genus $g$ such that $g$ is the smallest. Such a quiver is called a topological quiver. Note that in this paper only oriented 
surfaces are considered since we will use Eulers Polyhedron Theorem (Theorem 5.1) which is about a quiver embedded into an oriented surface.

A quiver $\Gamma$ is called connected if the underlying set of $\Gamma$ is connected. The set $F$ of faces of $\Gamma$ is the set of connected components of $S \backslash \Gamma$, or more precisely the complement of the underlying set of $\Gamma$ in $S$.

When the genus $g$ is zero, we can take the oriented surface $S$ to be the Riemann sphere, through the stereographic projection from the Riemann sphere to $\mathbb{R}^{2}$.

4.1.1. Combinatorial derivations from a quiver. We put together various combinatorially defined derivations on the path algebra of a quiver $\Gamma=(V, E)$. We recall the following notations.

(a) For $v \in V$, we call $D_{v}$ a vertex derivation and let $\mathfrak{D}_{V}$ denote the linear space spanned by $\left\{D_{v} \mid v \in V\right\}$, called the space of vertex derivations.

(b) For $p \in E$, we call $D_{p, p}$ an edge derivation and let $\mathfrak{D}_{E}$ denote the linear space spanned by $\left\{D_{p, p} \mid p \in E\right\}$, called the space of edge derivations.

For a face $f \in F$, let $\mathfrak{c}_{f}$ be the boundary of $f$. It is an unoriented cycle of $\Gamma$ consisting of arrows that are not necessarily in one direction, called a primitive cycle of $\Gamma$. Thus the set $C:=C_{\Gamma}$ of primitive cycles of $\Gamma$ is in bijection with the set $F$ of faces of $\Gamma$. For a primitive cycle $\mathfrak{c}=\mathfrak{c}_{f}$ of a face $f$, we will define a face derivation $D_{\mathfrak{c}}:=D_{\mathfrak{c}_{f}}$ on $\mathrm{k} \Gamma$. First define

$$
D_{\mathfrak{c}}(v)=0, v \in V ; \quad D_{\mathfrak{c}}(p)= \begin{cases}p, & p \text { is clockwise on } \mathfrak{c} \\ -p, & p \text { is counterclockwise on } \mathfrak{c}, \quad p \in E \\ 0, & p \text { is not on } \mathfrak{c}\end{cases}
$$

Here being clockwise or counterclockwise is viewed from inside the face for the primitive cycle. We then expand $D_{\mathfrak{c}}$ to $\mathrm{k} \Gamma$ by the product rule, noting that $\mathrm{k} \Gamma$ is the algebra generated by $V \cup E$.

If a cycle is shared by two faces, such as in the quiver of an oriented loop, there will be two face derivations from the two faces. Also, if $p$ is an edge in the interior of $\mathfrak{c}$, then $D_{\mathfrak{c}}(p)=0$. The name face derivation is justified by the following alternative description of $D_{\mathfrak{c}}$.

Lemma 4.1. Suppose a primitive cycle $\mathfrak{c} \in C_{\Gamma}$ is comprised of an ordered list of arrows $p_{1}, \cdots, p_{s} \in E$. Then

$$
D_{\mathfrak{c}}= \pm D_{p_{1}, p_{1}} \pm \cdots \pm D_{p_{s}, p_{s}}
$$

where $a \pm D_{p_{i}, p_{i}}$ is $D_{p_{i}, p_{i}}$ if $p_{i}$ is in clockwise direction when viewed from the interior of the face of $\mathfrak{c}$ and is $-D_{p_{i}, p_{i}}$ otherwise. In particular, $D_{\mathfrak{c}}$ is a derivation.

Proof. We only need to check that the two operators agree on $V \cup E$. But this is clear from the definition of $D_{\mathfrak{c}}$. tions.

Let $\mathfrak{D}_{F}$ denote the linear span of $\left\{D_{\mathfrak{c}_{f}} \mid f \in F\right\}$, called the space of face deriva- 
Since the concept of a face derivation will be important for the rest of the paper, we make the following remarks and illustrate their contents by the following quiver $\Gamma$.

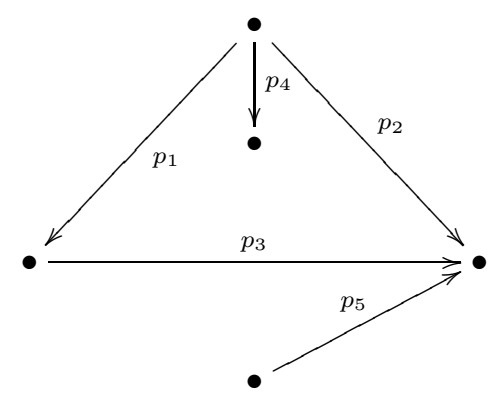

The quiver $\Gamma$ has two primitive cycles: the cycle $\mathfrak{c}_{1}$ of the finite face of $\Gamma$ and the cycle $\mathfrak{c}_{0}$ of the infinite face of $\Gamma$.

REMARK 4.2.

(a) In the case a quiver $\Gamma$ is planar, for the boundary $\mathfrak{c}_{0}$ of the infinite face, an arrow on $\mathfrak{c}_{0}$ is in clockwise direction when viewed from the interior of the infinite face means that the arrow is in counterclockwise direction when viewed from the interior of the quiver. For the quiver in the diagram (37), the arrow $p_{2}$ is clockwise for the primitive cycle from the finite face, but is counterclockwise for the primitive cycle from the infinite face.

(b) If an arrow $p$ is on the boundary of two faces of $\Gamma$, then $p$ will be in the clockwise direction on one boundary and in the counterclockwise direction on the other. Thus $D_{p, p}$ will have a plus sign in $D_{\mathfrak{c}}$ for the primitive cycle $\mathfrak{c}$ of one boundary and will have a minus sign in $D_{\mathfrak{c}}$ for the other. For our example of $\Gamma, D_{p_{1}, p_{1}}$ has a minus sign in $\mathfrak{c}_{1}$ and a plus sign in $\mathfrak{c}_{0}$.

(c) If an arrow $p$ is not on the boundary of two faces of $\Gamma$, then both sides of the arrow are in the same face of $\Gamma$. In other words, $p$ will appear twice in $D_{\mathfrak{c}}$ for the primitive cycle $\mathfrak{c}$ of this face, once in the clockwise direction, once in the counterclockwise direct. As a result, $D_{p, p}$ will appear exactly twice in $D_{\mathfrak{c}}$, once with a positive sign and once with a negative sign. Consequently, there will be no contribution of $D_{p, p}$ in $D_{\mathfrak{c}}$. For our example of $\Gamma$, both sides of $p_{4}$ are in the finite face of $\Gamma$. The primitive cycle $\mathfrak{c}_{1}$ of this face gives

$D_{\mathfrak{c}_{1}}=-D_{p_{1}, p_{1}}+D_{p_{4}, p_{4}}-D_{p_{4}, p_{4}}+D_{p_{2}, p_{2}}-D_{p_{3}, p_{3}}=-D_{p_{1}, p_{1}}+D_{p_{2}, p_{2}}-D_{p_{3}, p_{3}}$.

So $D_{p_{4}, p_{4}}$ does not contribute to $D_{\mathfrak{c}_{1}}$. Likewise, $D_{p_{5}, p_{5}}$ does not contribute to $D_{\mathfrak{c}_{0}}$ from the infinite face.

(d) The previous remark applies in particular when a quiver contains only a unique primitive cycle $\mathfrak{c}_{0}$ (in the case the quiver is planar, the unique primitive cycle is just the boundary of the infinite face, which is not proper). This is because for such a quiver, no arrow can appear on the boundary of two faces. Thus for such a quiver, we have $D_{\mathfrak{c}_{0}}=0$.

(e) By Remark 4.2.(b) and (c), each arrow $p \in E$ will appear in exactly one $D_{\mathfrak{c}}$ with a plus sign and in exactly another (or the same) $D_{\mathfrak{c}}$ with a minus sign. Consequently, we have

$$
\sum_{i=1}^{\gamma_{2}} D_{\mathfrak{c}_{i}}=0 .
$$


(f) For an oriented cycle $q$ of $\Gamma$, let $\mathfrak{c}$ be the corresponding primitive cycle. Then the $D_{\mathfrak{c}}$ defined here is different with $D_{q}$ given in Eq. (35).

4.1.2. Relation matrices. Denote $\gamma_{0}=|V|, \gamma_{1}=|E|, \gamma_{2}=|F|=|C|$. They are all finite since $\Gamma$ is finite. With these notations, we will use the following enumerations of sets.

$$
V=\left\{v_{i} \mid 1 \leq i \leq \gamma_{0}\right\}, \quad E=\left\{p_{k} \mid 1 \leq k \leq \gamma_{1}\right\}, \quad C=\left\{\mathfrak{c}_{j} \mid 1 \leq j \leq \gamma_{2}\right\}
$$

When $\gamma_{2}=1$, there are only a face and a primitive cycle in $\Gamma$. In this case, the underlying non-oriented graph of $\Gamma$ is a tree.

By Eq. (35) and Eq. (36), we have the following system of linear relations.

$$
\begin{aligned}
& D_{v_{i}}=\sum_{k=1}^{\gamma_{1}} c_{i, k} D_{p_{k}, p_{k}}=\sum_{p \in E, t(p)=v_{i}} D_{p, p}-\sum_{r \in E, h(r)=v_{i}} D_{r, r}, \quad 1 \leq i \leq \gamma_{0}, \\
& D_{\mathfrak{c}_{j}}=\sum_{k=1}^{\gamma_{1}} c_{\gamma_{0}+1+j, k} D_{p_{k}, p_{k}}=\sum_{k=1}^{\gamma_{1}} d_{j, k} D_{p_{k}, p_{k}}, \quad 1 \leq j \leq \gamma_{2} .
\end{aligned}
$$

where all $d_{j, k}= \pm 1$, or $=0$. Thus by Lemma 4.1 , we find that $\mathfrak{D}_{F}$ is a subspace of $\mathfrak{D}_{E}$.

\section{DEFINITION 4.3.}

(a) Define the (differential) vertex-arrow matrix $C_{v a}$ of $\Gamma$ to be the coefficient matrix of the linear system in Eq. (40).

(b) Define the (differential) cycle-arrow matrix $C_{c a}$ of $\Gamma$ to be the coefficient matrix of the linear system in Eq. (41).

(c) Define the connection matrix of $\Gamma$ to be the coefficient matrix $C_{\Gamma}$ of the combined linear system in Eq. (40) and Eq. (41), that is, the $\left(\gamma_{0}+\gamma_{2}\right) \times \gamma_{1^{-}}$ matrix $\left[\begin{array}{l}C_{v a} \\ C_{c a}\end{array}\right]$.

(d) Define the boundary matrix of a quiver $\Gamma$ to be the $\gamma_{2} \times \gamma_{2}$ matrix $B_{\Gamma}=$ $\left[e_{j, r}\right]_{0 \leq j, r \leq \gamma_{2}-1}$ in which $e_{j, j}$ is the number of arrows on $\mathfrak{c}_{j}$ that are also on $\mathfrak{c}_{r}$ for some $r \neq j$, and $-e_{j, r}$ for $r \neq j$ is the number of arrows on $\mathfrak{c}_{j}$ that are also on $\mathfrak{c}_{r}$.

REMARK 4.4.

(a) The matrix $C_{v a}$ encodes the relationship between the vertex derivations $D_{v_{i}}$, $1 \leq i \leq \gamma_{0}$ and the edge derivations $D_{p_{j}, p_{j}}, 1 \leq j \leq \gamma_{1}$. Since the edge derivations $D_{p_{k}, p_{k}}, 1 \leq k \leq \gamma_{1}$, are linearly independent the rank of the row space of $C_{v a}$ is $\operatorname{dim} \mathfrak{D}_{V}$.

(b) The matrix $C_{c a}$ encodes the relationship between the primitive cycle derivations $D_{\mathfrak{c}_{k}}, 0 \leq k \leq \gamma_{2}-1$ and the arrow derivations $D_{p_{j}, p_{j}}, 1 \leq j \leq \gamma_{1}$. The rank of the row space of $C_{c a}$ is just $\operatorname{dim} \mathfrak{D}_{F}$.

(c) By the definition of $e_{j, j}$ and $e_{j, r}$ for $r \neq j$, the matrix $B_{\Gamma}$ is independent of the direction of the quiver $\Gamma$ and only depends on the underlying graph.

In order to study the rank of the boundary matrix, we recall preparatory concepts and results of matrices. 


\section{DEFINITION 4.5.}

(a) A square matrix $M$ over a number field is called irreducible if $M$ cannot be written as a block matrix $M=\left[\begin{array}{cc}M_{1} & O \\ M_{3} & M_{2}\end{array}\right]$ where $O$ is a zero matrix and $M_{1}$ and $M_{2}$ are both square matrices.

(b) An $n \times n$-matrix $M=\left[m_{i, j}\right]$ is called weakly diagonally dominant if $\left|m_{i, i}\right| \geq \sum_{j=1, j \neq i}^{n}\left|m_{i, j}\right|$ for $i=1, \cdots, n$, with strict inequality for at least one $i$.

TheOREM 4.6. ([14, Section 10.7]) Let $M=\left[m_{i j}\right]$ be an $n \times n$ irreducible matrix over a number field. If $M$ is weakly diagonally dominant, then $M$ is invertible.

Using of this fact, we prove the following

LEMMA 4.7. Let $M=\left[m_{i j}\right]$ be an $n \times n$ irreducible matrix with entries in a number field. If, for each $i=1, \cdots, n, \sum_{j=1}^{n} m_{i, j}=0, m_{i, i}>0$ and $m_{i, j} \leq 0$ for $j \neq i$, then the rank of $M$ is $n-1$.

Proof. Since $\sum_{j=1}^{n} m_{i, j}=0$ for each $i=1, \cdots, n$, the sum of the $n$ column vectors of $M$ is zero. Thus the rank of $M$ is less than or equal to $n-1$.

On the other hand, consider the $(n-1) \times(n-1)$-submatrix $M_{1}$ consisting of the first $n-1$ rows and columns of $M$, that is $M_{1}=\left[m_{i, j}\right]_{1 \leq i, j \leq n-1}$. Since $\sum_{j=1}^{n} m_{i, j}=0$ for each $i=1, \cdots, n-1$, we have

$$
m_{i, i}=-\sum_{j=1, j \neq i}^{n} m_{i, j}=-\sum_{j=1, j \neq i}^{n-1} m_{i, j}-m_{i, n} \geq-\sum_{j=1, j \neq i}^{n-1} m_{i, j}=\sum_{j=1, j \neq i}^{n-1}\left|m_{i, j}\right| .
$$

Since $M$ is irreducible and symmetric, there is at least one $m_{i_{0}, n} \neq 0$ in the last column of $M$ other than $m_{n, n}$. Otherwise we would have $M=\left[\begin{array}{cc}M_{1} & \overrightarrow{0} \\ M_{0} & m_{n, n}\end{array}\right]$ for the zero vector $\overrightarrow{0}$, contradicting the irreducibility condition on $M$. Then, since $m_{i, j} \leq 0$ for $i \neq j$ by assumption, we have $m_{i_{0}, n}<0$. Therefore,

$$
m_{i_{0}, i_{0}}=-\sum_{j=1, j \neq i_{0}}^{n-1} m_{i_{0}, j}-m_{i_{0}, n}>\sum_{j=1, j \neq i_{0}}^{n-1}\left|m_{i_{0}, j}\right| .
$$

Thus, $M_{1}$ is a weakly diagonally dominant matrix. Then by Theorem $4.6, M_{1}$ is invertible. Together with the observation made at the beginning of the proof, we conclude that $r k(M)=n-1$.

\subsubsection{Ranks of relation matrices.}

THEOREM 4.8. Let $\Gamma$ be a connected finite quiver with no loops. Then the following statements hold:

(a) The vertex-arrow matrix $C_{v a}$ has rank $\gamma_{0}-1$.

(b) The cycle-arrow matrix $C_{c a}$ has rank $\gamma_{2}-1$.

(c) Suppose that the ground field $\mathbf{k}$ has characteristic 0 . Then the rank of the boundary matrix $B_{\Gamma}$ is $\gamma_{2}-1$.

Proof. (a) Since $e=\sum_{i=1}^{\gamma_{0}} v_{i}$ is the identity of $\mathrm{k} \Gamma$, we have

$$
D_{e}=\sum_{i=1}^{\gamma_{0}} D_{v_{i}}=0
$$


If $\gamma_{0}=1$, then $V=\left\{v_{1}\right\}$ and $D_{v_{1}}=0$ by Eq. (42). Thus $C_{v a}=0$ and its rank is $0=\gamma_{0}-1$.

If $\gamma_{0} \geq 2$, then by Eq. (42), $D_{v_{1}}$ is a linear combination of $D_{v_{i}}, 2 \leq i \leq \gamma_{0}$. Thus $\operatorname{rk}(G) \leq \gamma_{0}-1$. So we just need to prove $\operatorname{rk}\left(C_{v a}\right) \geq \gamma_{0}-1$. We will show this by induction on $\gamma_{0} \geq 2$.

First assume $\gamma_{0}=2$. Since $\Gamma$ is connected, by Eq. (40) the rows of $C_{v a}$ are non-zero. Then $\operatorname{rk}\left(C_{v a}\right) \geq 1$, as needed.

Next assume that the statement holds when $\gamma_{0}=n$ for $n \geq 2$ and consider a connected acyclic quiver $\Gamma$ with $\gamma_{0}=n+1$. By first listing the arrows $p_{1}, \cdots, p_{r}$ of $\Gamma$ that are connected to $v_{1}$, we see that $C_{v a}$ is a block matrix of the form

$$
C_{v a}=\left[\begin{array}{cc}
\vec{e} & \overrightarrow{0} \\
B & \vec{G}
\end{array}\right]
$$

where $\vec{e}$ is a row vector of dimension $r$ with entries $\pm 1, \overrightarrow{0}$ is a zero row vector of dimension $\gamma_{1}-r, B$ is a $\left(\gamma_{0} \times r\right)$-matrix and $\bar{G}$ is in fact the vertex-arrow matrix $G_{\bar{\Gamma}}$ of the quiver $\bar{\Gamma}$ obtained by deleting the vertex $v_{1}$ and its attached arrows from $\Gamma$. Since $\bar{\Gamma}$ has $n$ vertices, by the induction hypothesis, the rank of $\bar{G}$ is at least $n-1$. Thus there is a non-singular submatrix $\bar{H}$ of $\bar{G}$ of size $(n-1) \times(n-1)$. Adding back the first row and first column of $G$ to this submatrix $\bar{H}$, we obtain a submatrix $H$ of $C_{v a}$ of size $n \times n$. Since the added first row is $( \pm 1,0, \cdots, 0)$, the added first column is not a linear combination of the other columns in $H$. Thus $H$ is non-singular and the rank of $C_{v a}$ is at least $n$. This completes the induction and hence the proof of Item (a).

(b) We prove by induction on $\gamma_{2}$, which is also the number of rows of $C_{c a}$.

When $\gamma_{2}=1, \mathfrak{c}_{0}$ is the unique primitive cycle. In this case, $\Gamma$ is topologically homeomorphic to a point in the oriented surface $S$. By Remark 4.2(d), we have $D_{\mathfrak{c}_{1}}=0$. Thus, the unique row of $C_{c a}$ is zero. So, $\operatorname{rk}\left(C_{c a}\right)=0=\gamma_{2}-1$.

When $\gamma_{2}=2$, the rows of $C_{c a}$ are non-zero by definition and the sum of the only two rows of $C_{c a}$ is zero by Eq. (38). Hence, $\operatorname{rk}\left(C_{c a}\right)=1=\gamma_{2}-1$.

Assume that the statement is verified when $\gamma_{2}=n \geq 2$ and consider $\Gamma$ with $\gamma_{2}=n+1$. By reordering the arrows $p_{1}, \cdots, p_{\gamma_{1}}$ of $\Gamma$ if necessary, we can assume that the arrows of $\mathfrak{c}_{1}$ are $p_{1}, \cdots, p_{s}$ where $s \geq 2$ since $\Gamma$ has not loops. Thus the coefficients $c_{\gamma_{0}+1,1}, c_{\gamma_{0}+1,2}, \cdots, c_{\gamma_{0}+1, \gamma_{1}}$ of $\mathfrak{c}_{1}$ in Eq. (41) satisfy that $c_{\gamma_{0}+1,1}, \cdots, c_{\gamma_{0}+1, s}$ are all \pm 1 and $c_{\gamma_{0}+1, s+1}=\cdots=c_{\gamma_{0}+1, \gamma_{1}}=0$. Then, since the sum of the row vectors of $C_{c a}$ is zero by Eq. (38), by reordering the primitive cycles $\mathfrak{c}_{1}, \cdots, \mathfrak{c}_{n}$ if necessary, we can also assume that the first coefficient of $\mathfrak{c}_{1}$ satisfies $c_{\gamma_{0}+2,1}=-c_{\gamma_{0}+1,1}$. Thus, there is $r$ between 1 and $s$ such that $c_{\gamma_{0}+2, i}=-c_{\gamma_{0}+1, i}$ for $i=1, \cdots, r$ and $c_{\gamma_{0}+2, j}=0$ for $j=r+1, \cdots, s$. Then the first two rows of $C_{c a}$ are of the form

$$
\left[\begin{array}{ccc} 
\pm 1, \cdots, \pm 1, & \pm 1, \cdots, \pm 1, & 0, \cdots, 0 \\
\underbrace{\mp 1, \cdots, \mp 1}_{r \text { terms }}, & \underbrace{0, \cdots, 0}_{s-r \text { terms }}, & \underbrace{*, \cdots, *}_{\gamma_{1}-r \text { terms }}
\end{array}\right] .
$$

Here the signs in the second row for the first $r$ terms are opposite to the signs in the corresponding terms in the first row. Thus, the matrix $C_{c a}$ has the form

$$
C_{c a}=\left[\begin{array}{cc}
\vec{a}_{1} & \vec{b}_{1} \\
-\vec{a}_{1} & \vec{b}_{2} \\
O & \bar{K}
\end{array}\right]
$$


where $\vec{a}_{1}$ is a row vector of dimension $r$ with entries $\pm 1, O$ is a zero matrix of size $(n-1) \times r, \vec{b}_{1}, \vec{b}_{2}$ are row vectors of dimension $\gamma_{1}-r$ and $\bar{K}$ is a matrix of size $(n-1) \times\left(\gamma_{1}-r\right)$. We can write

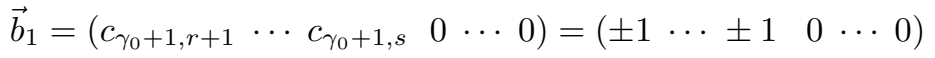

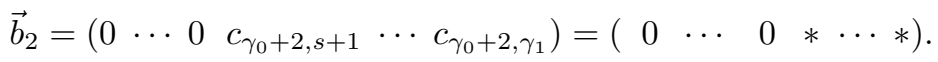

Deleting the arrows $p_{1}, \cdots, p_{r}$, we get a quiver $\bar{\Gamma}$ with $n$ primitive cycles $\mathfrak{c}_{1}^{\prime}, \mathfrak{c}_{3}, \cdots, \mathfrak{c}_{n}$ where $\mathfrak{c}_{1}^{\prime}$ is obtained via amalgamating $\mathfrak{c}_{1}$ and $\mathfrak{c}_{2}$. Moreover, with

$$
\vec{b}:=\vec{b}_{1}+\vec{b}_{2}=\left(c_{\gamma_{0}+1, r+1} \cdots c_{\gamma_{0}+1, s} c_{\gamma_{0}+2, s+1} \cdots c_{\gamma_{0}+2, \gamma_{1}}\right),
$$

we find that the cycle-arrow matrix $C_{c a, \bar{\Gamma}}$ of the quiver $\bar{\Gamma}$ is just $\left[\frac{\vec{b}}{K}\right]$.

By the induction hypothesis, $\operatorname{rk}\left(C_{c a, \bar{\Gamma}}\right)=n-1$. By Eq. (38), $-\vec{b}$ is the sum of all rows of $\bar{K}$. Hence, $\operatorname{rk}(\bar{K})=n-1$, that is, $\bar{K}$ has full row rank. Since $r \geq 1$, the second row $\left(-\vec{e}_{1} \vec{b}_{2}\right)$ of $C_{c a}$ is linearly independent from the last $n-1$ rows of $C_{c a}$. So, $\operatorname{rk}\left(C_{c a}\right) \geq(n-1)+1=n$. By Eq. (38), the row vectors of $C_{c a}$ are linearly dependent. Therefore, $\operatorname{rk}\left(C_{c a}\right)=n=\gamma_{2}-1$, completing the induction.

(c) By definition, $B_{\Gamma}$ is a $\left(\gamma_{2} \times \gamma_{2}\right)$-matrix.

In the special case of $\gamma_{2}=1$, there are no cycles on $\Gamma$ except the boundary $\mathfrak{c}_{1}$ for the unique face. So all the arrows are on $\mathfrak{c}_{1}$ only. Thus $B_{\Gamma}=0$ and hence its rank is 0 .

For the case when $\gamma_{2}>1$, we apply Lemma 4.7. For this we just need to verify that $B_{\Gamma}$ satisfies the conditions for $M$ in the lemma as follows.

- By definition, $B_{\Gamma}$ has entries in $\mathbb{Z}$ and hence in a number field, say $\mathbb{Q}$.

- By Definition 4.3 on $e_{i, j}, B_{\Gamma}$ is symmetric and $e_{i, j} \leq 0$ for $i \neq j$.

- $B_{\Gamma}$ is irreducible since $\Gamma$ is connected.

- Since $\gamma_{0}>0$, each cycle $\mathfrak{c}_{j}, 1 \leq j \leq \gamma_{2}$ shares at least one arrow with the cycle of a neighboring face. Thus $e_{j, j}>0$.

- By the definition of $e_{j, r}, 1 \leq j, r \leq \gamma_{2}$, the sum of the entries of each row of $B_{\Gamma}$ is zero. Thus the sum of the columns of $B_{\Gamma}$ is zero. Since $B_{\Gamma}$ is symmetric, the sum of the rows of $B_{\Gamma}$ is also zero.

Thus Item (c) is proved.

4.2. Dimensions of combinatorial derivations. From Theorem 4.8, we have the following dimensional formulas of combinatorial derivations.

THEOREM 4.9. Let $\Gamma$ be a connected finite acyclic quiver. Then,

(a) $\operatorname{dim} \mathfrak{D}_{V}=|V|-1$;

(b) $\operatorname{dim} \mathfrak{D}_{F}=|F|-1$;

(c) $\mathfrak{D}_{V}$ and $\mathfrak{D}_{F}$ are linearly disjoint subspaces of $\mathfrak{D}_{E}$.

The following is a direct consequence of Theorem 4.9. More applications of the theorem will be given in Section 5 .

Corollary 4.10. The dimensions of the spaces of derivations $\mathfrak{D}_{V}, \mathfrak{D}_{E}$ and $\mathfrak{D}_{F}$ of a connected acyclic quiver $\Gamma$ only depend on the underlying graph of $\Gamma$ and not depend on the choice of orientations of the edges. 
Proof. Item (a) follow directly from of Theorem 4.8.(a) by Eq. (40). Similarly, Item (b) follows directly from Theorem 4.8.(b) by Eq. (41). So we just need to prove Item (c).

Let $D$ be in $\mathfrak{D}_{V} \cap \mathfrak{D}_{F}$. Then there are constants $a_{i}, 1 \leq i \leq \gamma_{0}$, and $b_{j}, 1 \leq j \leq \gamma_{2}$, in $\mathbf{k}$ such that

$$
\sum_{i=1}^{\gamma_{0}} a_{i} D_{v_{i}}=D=\sum_{j=1}^{\gamma_{2}} b_{j} D_{\mathfrak{c}_{j}}
$$

To prove Proposition 4.9.(c), we just need to show $D=0$.

We first rewrite the left sum in Eq. (43). By Eq. (40), we obtain

$$
\sum_{i=1}^{\gamma_{0}} a_{i} D_{v_{i}}=\sum_{i=1}^{\gamma_{0}} a_{i} \sum_{k=1}^{\gamma_{1}} c_{i, k} D_{p_{k}, p_{k}}=\sum_{k=1}^{\gamma_{1}}\left(\sum_{i=1}^{\gamma_{0}} c_{i, k} a_{i}\right) D_{p_{k}, p_{k}} .
$$

Since by definition, $c_{i, k}= \begin{cases}1, & t\left(p_{k}\right)=v_{i}, \\ -1, & h\left(p_{k}\right)=v_{i}, \\ 0, & \text { otherwise, }\end{cases}$

$$
\sum_{i=1}^{\gamma_{0}} a_{i} D_{v_{i}}=\sum_{k=1}^{\gamma_{1}}\left(a_{t\left(p_{k}\right)}-a_{h\left(p_{k}\right)}\right) D_{p_{k}, p_{k}}
$$

On the other hand, by Eq. (41), the right sum of Eq. (43) can be written as

$$
\sum_{j=1}^{\gamma_{2}} b_{j} D_{\mathfrak{c}_{j}}=\sum_{j=1}^{\gamma_{2}} b_{j}\left(\sum_{k=1}^{\gamma_{1}} d_{j, k} D_{p_{k}, p_{k}}\right)=\sum_{k=1}^{\gamma_{1}}\left(\sum_{j=1}^{\gamma_{2}} b_{j} d_{j, k}\right) D_{p_{k}, p_{k}}
$$

Here by Eq. (36), $d_{j, k}= \begin{cases}1, & \text { if } p_{k} \text { is on } \mathfrak{c}_{j} \text { in clockwise direction, } \\ -1, & \text { if } p_{k} \text { is on } \mathfrak{c}_{j} \text { in counter clockwise direction, } \\ 0, & \text { if } p_{k} \text { is not on } \mathfrak{c}_{j} .\end{cases}$

By Remark 4.2.(e), any given arrow $p_{k}$ appears twice on the boundaries of the cycles $\mathfrak{c}_{j}, 1 \leq j \leq \gamma_{2}$, once in clockwise direction and once in counter clockwise direction. Let $1 \leq x\left(p_{k}\right) \leq \gamma_{2}$ (resp. $1 \leq y\left(p_{k}\right) \leq \gamma_{2}$ ) denote the label of the cycle containing $p_{k}$ in the clockwise (resp. counter clockwise) direction. It is possible that $x\left(p_{k}\right)=y\left(p_{k}\right)$. Then we have $d_{x\left(p_{k}\right), k}=1$ and $d_{y\left(p_{k}\right), k}=-1$. Then Eq. (45) becomes

$$
\sum_{j=1}^{\gamma_{2}} b_{j} D_{\mathfrak{c}_{j}}=\sum_{k=1}^{\gamma_{1}}\left(b_{x\left(p_{k}\right)}-b_{y\left(p_{k}\right)}\right) D_{p_{k}, p_{k}}
$$

Thus by Eq. (44) and (46), we find that Eq. (43) is equivalent to

$$
D=\sum_{k=1}^{\gamma_{1}}\left(a_{t\left(p_{k}\right)}-a_{h\left(p_{k}\right)}\right) D_{p_{k}, p_{k}}=\sum_{k=1}^{\gamma_{1}}\left(b_{x\left(p_{k}\right)}-b_{y\left(p_{k}\right)}\right) D_{p_{k}, p_{k}} .
$$

Since the set $\left\{D_{p_{k}, p_{k}} \mid 1 \leq k \leq \gamma_{1}\right\}$ of derivations is linearly independent, we thus obtain the following system of linear equations:

$$
a_{t\left(p_{k}\right)}-a_{h\left(p_{k}\right)}=b_{x\left(p_{k}\right)}-b_{y\left(p_{k}\right)}, \quad 1 \leq k \leq \gamma_{1} .
$$


Lemma 4.11. For $1 \leq k \leq \gamma_{1}$, a change of the direction of $p_{k}$ leads to a change of signs on both sides of the $k$-th equation in Eq. (48), yielding an equivalent equation.

Proof. Let $\Gamma^{\prime}$ be the quiver with $p_{k}$ replaced by $q_{k}$ in the opposite direction. Then $t\left(p_{k}\right)=h\left(q_{k}\right)$ and $h\left(p_{k}\right)=t\left(q_{k}\right)$. Then the left hand side of Eq. (48) becomes

$$
a_{h\left(q_{k}\right)}-a_{t\left(q_{k}\right)}=-\left(a_{t\left(q_{k}\right)}-a_{h\left(q_{k}\right)}\right) .
$$

Likewise, by the definition of $x\left(p_{k}\right)$ and $y\left(p_{k}\right)$ we have $x\left(q_{k}\right)=y\left(p_{k}\right)$ and $y\left(q_{k}\right)=$ $x\left(p_{k}\right)$. This reverses the sign on the right hand side of Eq. (48).

LEMma 4.12. Any set of solutions $b_{j}, 1 \leq j \leq \gamma_{2}$, in the system Eq. (48) satisfies the following system of linear equations:

$$
e_{j, j} b_{j}+\sum_{1 \leq r \leq \gamma_{2}, r \neq j} e_{j, r} b_{r}=0, \quad 1 \leq j \leq \gamma_{2}
$$

where $e_{i, j}$ is defined in Definition 4.3 .

Proof. Fix a $1 \leq j \leq \gamma_{2}$. Let $p_{k_{1}}, \cdots, p_{k_{s}}$ be the arrows on $\mathfrak{c}_{j}$ that are also on $\mathfrak{c}_{r}$ for some $r \neq j$, that is, these are the arrows on $\mathfrak{c}_{j}$ that are not in the interior of $\mathfrak{c}_{j}$. Because of Lemma 4.11, we can change the directions of some of the arrows $p_{k_{1}}, \cdots, p_{k_{s}}$ so that all the arrows in $\mathfrak{c}_{j}$ go clockwise, without changing the solution set of the system in Eq. (48). After this is done, when we add the $k_{\ell}$-th equations

$$
a_{t\left(p_{k_{\ell}}\right)}-a_{h\left(p_{k_{\ell}}\right)}=b_{x\left(p_{k_{\ell}}\right)}-b_{y\left(p_{k_{\ell}}\right)}, \quad 1 \leq \ell \leq s,
$$

to the system (48), the left hand sides add up to zero. For the right hand side, we have $x\left(p_{k_{\ell}}\right)=j, 1 \leq \ell \leq s$, and $y\left(p_{k_{\ell}}\right)$ is the label of the other cycle that $p_{k_{\ell}}$ is on. Note that $s=e_{j, j}$ by definition. Thus the right hand sides add up to

$$
e_{j, j} b_{j}+\sum_{1 \leq r \leq \gamma_{2}, r \neq j} e_{j, r} b_{r}
$$

for $e_{j, j}$ and $e_{j, r}$ as defined in the lemma. This finishes the proof.

We can now complete the proof of Theorem 4.9.(c) as follows. Let $D$ be in $\mathfrak{D}_{V} \cap \mathfrak{D}_{F}$. Then there are $a_{i}, 1 \leq i \leq \gamma_{0}$ and $b_{j}, 1 \leq j \leq \gamma_{2}$ such that they satisfy Eq. (43). Then they satisfy the linear system in Eq. (48). Then by Lemma $4.12, b_{j}$ satisfies the system of linear equations in Eq. (49). Since the coefficient matrix of this system is the boundary matrix $B_{\Gamma}$ by Lemma 4.12 and hence has rank $\gamma_{2}-1$ by Theorem 4.8.(c), the system in Eq. (49) has unique nonzero solutions $b_{i}$ up a constant. But the choice of $b_{x\left(p_{k}\right)}=b_{y\left(p_{k}\right)}, 1 \leq k \leq \gamma_{1}$ together with $a_{t\left(p_{k}\right)}=a_{h\left(p_{k}\right)}, 1 \leq i \leq \gamma_{0}$ is already a nonzero solution of Eq. (48) and hence of Eq. (49). Thus this gives the unique solution of Eq. (48). Hence by Eq. (47), we have $D=0$. Therefore $\mathfrak{D}_{V} \cap \mathfrak{D}_{F}=0$, showing that $\mathfrak{D}_{V}$ and $\mathfrak{D}_{F}$ are linearly disjoint.

Now the proof of Theorem 4.9(c) is completed.

5. Euler's formula and Hochschild cohomology. In this section we give two applications of the dimensional formulas of combinatorial derivations in Theorem 4.9. We first present a differential enrichment of Euler's Polyhedron Theorem (Theorem 5.1) by showing that the numerical relation in Euler's formula among the geometric objects of vertices, edges and faces of the underlying graph of a quiver comes from an algebraic relation among the spaces of derivations associated to these geometric objects. 
5.1. Euler's polyhedron formula from a differential point of view. We recall the following classical result from [4] and [8].

Theorem 5.1. (Euler's Polyhedron Theorem) For any connected quiver $\Gamma$ of genus $g$, we have

$$
|V|-|E|+|F|=2-2 g .
$$

Recall that the genus of a quiver $\Gamma$ is defined to be the smallest genus of an oriented surface in which the quiver can be embedded. This genus is used in Euler's Polyhedron Theorem (Theorem 5.1) by the theory of topological graphs [8]. Our results in this paper, e.g. Theorem 5.2 and Proposition 5.4, are based on this Euler's theorem and will use the same genus.

We show that there is a strengthening of Euler's numerical formula in the context of derivations.

Theorem 5.2. (Differential Formulation of Euler's Polyhedron Theorem) For a connected finite acyclic quiver $\Gamma$ of genus $g$, the spaces $\mathfrak{D}_{V}, \mathfrak{D}_{E}, \mathfrak{D}_{F}$ of vertex derivations, arrow derivations and face derivations satisfy the following relation.

$$
\operatorname{dim}_{\mathbf{k}} \mathfrak{D}_{E} /\left(\mathfrak{D}_{V} \oplus \mathfrak{D}_{F}\right)=2 g
$$

where the direct sum is the interior sum of subspaces.

In particular, in the case the genus $g=0$,

$$
\mathfrak{D}_{E}=\mathfrak{D}_{V} \oplus \mathfrak{D}_{F} .
$$

Proof. By Theorem 4.9, we have

$$
\operatorname{dim}\left(\mathfrak{D}_{V} \oplus \mathfrak{D}_{F}\right)=\operatorname{dim} \mathfrak{D}_{V}+\operatorname{dim} \mathfrak{D}_{F}=|V|-1+|F|-1 .
$$

But, $\operatorname{dim} \mathfrak{D}_{E}=|E|$. By Euler's formula in Theorem 5.1,

$$
\operatorname{dim} \mathfrak{D}_{E}-\operatorname{dim}\left(\mathfrak{D}_{V} \oplus \mathfrak{D}_{F}\right)=|E|-(|V|-1+|F|-1)=2 g .
$$

This gives Eq. (51) since $\mathfrak{D}_{V} \oplus \mathfrak{D}_{F}$ is a subspace of $\mathfrak{D}_{E}$.

REMARK 5.3. As we see above, Euler's theorem is used in the proof of Theorem 5.2. Conversely, the equation $\operatorname{dim}_{\mathbf{k}} \mathfrak{D}_{E} /\left(\mathfrak{D}_{V} \oplus \mathfrak{D}_{F}\right)=2 g$ gives Euler's formula by Eq. (53). Thus, as its name suggests, Theorem 5.2 gives a strengthened form of Euler's theorem from the view point of derivations. It would be interesting to find a proof of Theorem 5.2 without using Euler's theorem.

5.2. The Structure of Lie algebra $H H^{1}(\mathbf{k} \Gamma)$. We next apply Theorem 4.9 to study $H H^{1}(\mathbf{k} \Gamma)$. We first give a dimensional formula of $H H^{1}(\mathbf{k} \Gamma)$. Then we obtain a canonical basis of $H H^{1}(\mathbf{k} \Gamma)$. 


\subsubsection{A dimensional formula of $H H^{1}(\mathbf{k} \Gamma)$. Denote}

$$
\mathcal{P}_{L}:=\{s \in \mathcal{P} \mid h(s)=t(s)\}, \quad \mathfrak{B}_{L}:=\left\{D_{s} \mid s \in \mathcal{P}_{L}\right\}, \quad \mathfrak{D}_{L}:=\mathbf{k} \mathfrak{B}_{L}=\mathfrak{D}\left(\mathbf{k} \mathcal{P}_{L}\right) .
$$

Then on one hand we have the disjoint union $\mathcal{P}=\mathcal{P}_{A} \sqcup \mathcal{P}_{L}$, and hence $\mathbf{k} \Gamma=\mathbf{k} \mathcal{P}_{A} \oplus \mathbf{k} \mathcal{P}_{L}$. Since $\operatorname{ker} \mathfrak{D}=\mathbf{k} \subseteq \mathbf{k} \mathcal{P}_{L}$ for the linear map $\mathfrak{D}: \mathbf{k} \Gamma \rightarrow \operatorname{Der}(\mathbf{k} \Gamma)$ in Eq. (2), we have

$$
\operatorname{InDer}(\mathbf{k} \Gamma)=\mathfrak{D}(\mathbf{k} \Gamma) \cong \mathfrak{D}_{1} \oplus \mathfrak{D}_{L}
$$

On the other hand, since $\mathfrak{D}_{1} \subseteq \operatorname{InDer}(\mathbf{k} \Gamma)$ and $\operatorname{Der}(\mathbf{k} \Gamma)=\mathfrak{D}_{1} \oplus \mathfrak{D}_{2}$ from Theorem 3.4 , we have

$$
\operatorname{InDer}(\mathbf{k} \Gamma)=\operatorname{InDer}(\mathbf{k} \Gamma) \cap\left(\mathfrak{D}_{1} \oplus \mathfrak{D}_{2}\right)=\mathfrak{D}_{1} \oplus\left(\operatorname{InDer}(\mathbf{k} \Gamma) \cap \mathfrak{D}_{2}\right) .
$$

By Proposition 3.8, we have $\mathfrak{D}_{L} \subseteq \operatorname{InDer}(\mathbf{k} \Gamma) \cap \mathfrak{D}_{2}$. Thus from Eq. (54) and Eq. (55) we obtain

$$
\operatorname{InDer}(\mathbf{k} \Gamma) \cap \mathfrak{D}_{2}=\mathfrak{D}_{L}
$$

Therefore we have

$$
\begin{aligned}
H H^{1}(\mathbf{k} \Gamma) & =\operatorname{Der}(\mathbf{k} \Gamma) / \operatorname{InDer}(\mathbf{k} \Gamma) \\
& =\left(\operatorname{InDer}(\mathbf{k} \Gamma)+\mathfrak{D}_{2}\right) / \operatorname{InDer}(\mathbf{k} \Gamma) \\
& \cong \mathfrak{D}_{2} /\left(\operatorname{InDer}(\mathbf{k} \Gamma) \cap \mathfrak{D}_{2}\right) \\
& =\mathfrak{D}_{2} / \mathfrak{D}_{L},
\end{aligned}
$$

giving us the following commutative diagram of exact sequences of Lie algebras.

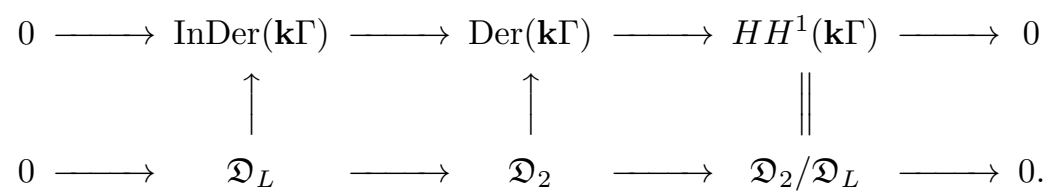

An almost oriented cycle in a quiver $\Gamma$ is defined to be a pair $(p, r)$ where $p \in E$ and $r \in \mathcal{P}$ with $r \neq p$ and $r \| p$. It is so namely since $p$ and $r$ form an oriented cycle by reversing the arrow $p$. Let $\Gamma_{A L}$ be the set of almost oriented cycles of $\Gamma$. Denote

$$
\mathfrak{B}_{E}:=\left\{D_{p, p} \mid p \in E\right\}, \quad \mathfrak{B}_{A L}:=\left\{D_{p, r} \mid(p, r) \in \Gamma_{A L}\right\}, \quad \mathfrak{D}_{A L}=\mathbf{k} \mathfrak{B}_{A L} .
$$

Then by the definition of $\mathfrak{B}_{2}$ in Eq. (28), we have the disjoint union

$$
\mathfrak{B}_{2}=\mathfrak{B}_{E} \sqcup \mathfrak{B}_{A L} \quad \text { and } \quad \mathfrak{D}_{2}=\mathfrak{D}_{E} \oplus \mathfrak{D}_{A L} .
$$

From this and Eq.(56), we obtain

$$
H H^{1}(\mathbf{k} \Gamma) \cong \mathfrak{D}_{2} / \mathfrak{D}_{V} \cong\left(\mathfrak{D}_{E} / \mathfrak{D}_{V}\right) \oplus \mathfrak{D}_{A L}
$$

Proposition 5.4. Let $\Gamma$ be a connected acyclic quiver. Then

$$
\operatorname{dim}_{\mathbf{k}} H H^{1}(\mathbf{k} \Gamma)=|F|+\left|\Gamma_{A L}\right|-1+2 g .
$$

In the case $g=0, H H^{1}(\mathbf{k} \Gamma) \neq 0$ if and only if $\Gamma$ contains an unoriented cycle. 
Proof. By Eq. (59), Theorem 4.8 and Euler's Theorem 5.1, we have $\operatorname{dim} H H^{1}(\mathbf{k} \Gamma)=\operatorname{dim} \mathfrak{D}_{E}-\operatorname{dim} \mathfrak{D}_{V}+\operatorname{dim} \mathfrak{D}_{A L}=|E|-|V|+1+\left|\Gamma_{A L}\right|=|F|+\left|\Gamma_{A L}\right|+2 g-1$.

Then the last statement follows easily.

Compare Proposition 5.4 with Happel's formula [11], $\operatorname{dim}_{\mathbf{k}} H H^{1}(\mathbf{k} \Gamma)=1-|V|+$ $\sum_{\alpha \in E} v(\alpha)$, where $v(\alpha)=\operatorname{dim}_{\mathbf{k}} t(\alpha) \mathbf{k} \Gamma h(\alpha)$. The two formulas can be easily derived from each other. Our formula makes it easy to guess a canonical basis of $H H^{1}(\mathbf{k} \Gamma)$. Indeed, verifying that this guess actually works is the motivation behind the introduction of the combinatorial derivations in Section 4.

5.2.2. A canonical basis of $H H^{1}(\mathbf{k} \Gamma)$. Proposition 5.4 suggests that a canonical basis of $H H^{1}(\mathbf{k} \Gamma)$ can be obtained from derivations defined from the faces (through their unoriented cycles) and $\Gamma_{A L}$. We show that this is indeed the case.

Denote

$$
\mathfrak{B}_{F}^{-}=\left\{D_{\mathfrak{c}_{i}} \mid 2 \leq i \leq \gamma_{2}\right\}
$$

then by Theorem 4.9.(b) and Eq. (38), we have $\mathfrak{D}_{F}=\mathbf{k} \mathfrak{B}_{F}^{-}$. Our main result on outer derivations is the following

Theorem 5.5. (Basis Theorem of the First Hochschild Cohomology) Let $\Gamma$ be a connected finite acyclic quiver over a ground field $\mathbf{k}$ of characteristic 0 . Then the disjoint union

$$
\mathfrak{B}_{A L} \sqcup \mathfrak{B}_{F}^{-} \sqcup \mathfrak{B}^{*}
$$

forms a basis of $H H^{1}(\mathbf{k} \Gamma)$, where $\mathfrak{B}^{*}$ is any basis of $\mathfrak{D}_{E} /\left(\mathfrak{D}_{V} \oplus \mathfrak{D}_{F}\right)$.

Proof. We have $\mathfrak{D}_{E} /\left(\mathfrak{D}_{V} \oplus \mathfrak{D}_{F}\right) \cong\left(\mathfrak{D}_{E} / \mathfrak{D}_{V}\right) /\left(\left(\mathfrak{D}_{V} \oplus \mathfrak{D}_{F}\right) / \mathfrak{D}_{V}\right)$. It follows that

$$
\begin{aligned}
\mathfrak{D}_{E} / \mathfrak{D}_{V} & \cong \mathfrak{D}_{E} /\left(\mathfrak{D}_{V} \oplus \mathfrak{D}_{F}\right) \oplus\left(\mathfrak{D}_{V} \oplus \mathfrak{D}_{F}\right) / \mathfrak{D}_{V} \\
& \cong \mathfrak{D}_{E} /\left(\mathfrak{D}_{V} \oplus \mathfrak{D}_{F}\right) \oplus \mathfrak{D}_{F} .
\end{aligned}
$$

Thus by Eq. (59), $H H^{1}(\mathbf{k} \Gamma) \cong\left(\mathfrak{D}_{E} / \mathfrak{D}_{V}\right) \oplus \mathfrak{D}_{A L} \cong \mathfrak{D}_{E} /\left(\mathfrak{D}_{V} \oplus \mathfrak{D}_{F}\right) \oplus \mathfrak{D}_{F} \oplus \mathfrak{D}_{A L}$.

$\mathfrak{D}_{F}$ has a basis $\mathfrak{B}_{F}^{-}$by Theorem 4.9.(b) and $\mathfrak{D}_{A L}$ has a basis $\mathfrak{B}_{A L}$. Since they are disjoint by Eq. (58), we obtain Theorem 5.5 for any basis $\mathfrak{B}^{*}$ of $\mathfrak{D}_{E} /\left(\mathfrak{D}_{V} \oplus \mathfrak{D}_{F}\right)$.

We have not found a canonical way to determine $\mathfrak{B}^{*}$ except when the genus is zero, see the following Corollary 5.6.

We require that $\mathbf{k}$ is of characteristic 0 in Theorem 5.5 because its proof depends on Theorem 4.6. When the genus $g$ is $0, \Gamma$ is a planar quiver. In this case, by Euler's theorem, $\operatorname{dim} \mathfrak{D}_{E} /\left(\mathfrak{D}_{V} \oplus \mathfrak{D}_{F}\right)=0$. Also, we can take $\mathfrak{c}_{1}$ to be the primitive cycle for the unique unbounded face. Thus we can make the choice of $\mathfrak{B}_{F}^{-}$as well as $\mathfrak{B}_{A L}$ completely canonical. Hence, we have

Corollary 5.6. (Basis Theorem for Planar Quiver) Let $\Gamma$ be a connected finite planar acyclic quiver and let the ground field $\mathbf{k}$ be of characteristic 0 . Then the disjoint union

$$
\mathfrak{B}_{A L} \cup\left\{D_{\mathfrak{c}} \mid \mathfrak{c} \text { is a bounded primitive cycle }\right\}
$$

forms a basis of $H H^{1}(\mathbf{k} \Gamma)$. 
5.2.3. Lie algebra structure of $H H^{1}(\mathbf{k} \Gamma)$. From the basis $\mathfrak{B}_{A L} \cup \mathfrak{B}_{F}^{-}$in Corollary 5.6, the structural constants of the Lie algebra $H H^{1}(\mathbf{k} \Gamma)$ can be computed explicitly by Eq. (33) since we have $\mathfrak{B}_{A L} \subseteq \mathfrak{B}_{2}$ and $\mathfrak{B}_{F} \subseteq \mathbf{k} \mathfrak{B}_{E}=\mathfrak{D}_{E} \subseteq \mathfrak{D}_{2}=\mathbf{k} \mathfrak{B}_{2}$. Concretely, for $D_{\mathfrak{c}}, D_{\mathfrak{q}} \in \mathfrak{B}_{F}$, let

$$
\begin{aligned}
& D_{\mathfrak{c}}=a_{1} D_{p_{1}, p_{1}}+a_{2} D_{p_{2}, p_{2}}+\cdots+a_{\gamma_{1}} D_{p_{\gamma_{1}}, p_{\gamma_{1}}}, \\
& D_{\mathfrak{q}}=b_{1} D_{p_{1}, p_{1}}+b_{2} D_{p_{2}, p_{2}}+\cdots+b_{\gamma_{1}} D_{p_{\gamma_{1}}, p_{\gamma_{1}}}
\end{aligned}
$$

for the arrow set $E=\left\{p_{1}, p_{2}, \cdots, p_{\gamma_{1}}\right\}$. Then by Eq. (33) we have

$$
\begin{aligned}
{\left[D_{\mathfrak{c}}, D_{\mathfrak{q}}\right] } & =\sum_{i, j=1}^{\gamma_{1}} a_{i} b_{j}\left[D_{p_{i}, p_{i}}, D_{p_{j}, p_{j}}\right] \\
& =\sum_{i, j=1}^{\gamma_{1}} a_{i} b_{j}\left(D_{p_{j}, D_{p_{i}, p_{i}}\left(p_{j}\right)}-D_{p_{i}, D_{p_{j}, p_{j}}\left(p_{i}\right)}\right) \\
& =\sum_{i=1}^{\gamma_{1}} a_{i} b_{i}\left(D_{p_{i}, p_{i}}-D_{p_{i}, p_{i}}\right) \\
& =0 .
\end{aligned}
$$

This means that $\mathfrak{D}_{F}=\mathbf{k} \mathfrak{B}_{F}$ is an abelian Lie sub-algebra of the Lie algebra $H H^{1}(\mathbf{k} \Gamma)$.

For $D_{r, s} \in \mathfrak{B}_{A L}$, by Eq. (33) we have

$$
\begin{aligned}
{\left[D_{r, s}, D_{\mathfrak{c}}\right]=} & a_{1}\left[D_{r, s}, D_{p_{1}, p_{1}}\right]+\cdots+a_{\gamma_{1}}\left[D_{r, s}, D_{p_{\gamma_{1}}, p_{\gamma_{1}}}\right] \\
= & a_{1}\left(D_{p_{1}, D_{r, s}\left(p_{1}\right)}-D_{r, D_{p_{1}, p_{1}}(s)}\right)+\cdots+a_{\gamma_{1}}\left(D_{p_{\gamma_{1}}, D_{r, s}\left(p_{\gamma_{1}}\right)}-D_{r, D_{p_{\gamma_{1}}, p_{\gamma_{1}}}(s)}\right) \\
= & \left(a_{1} D_{p_{1}, D_{r, s}\left(p_{1}\right)}+\cdots+a_{\gamma_{1}} D_{p_{\gamma_{1}}, D_{r, s}\left(p_{\gamma_{1}}\right)}\right) \\
& -\left(a_{1} D_{r, D_{p_{1}, p_{1}}(s)}+\cdots+a_{\gamma_{1}} D_{r, D_{p_{\gamma_{1}}, p_{\gamma_{1}}}(s)}\right) .
\end{aligned}
$$

Write $r=p_{i_{0}}$ for some $1 \leq i_{0} \leq \gamma_{1}$. Then $a_{1} D_{p_{1}, D_{r, s}\left(p_{1}\right)}+\cdots+a_{\gamma_{1}} D_{p_{\gamma_{1}}, D_{r, s}\left(p_{\gamma_{1}}\right)}=$ $a_{i_{0}} D_{r, s}$. Also, write $s=p_{i_{1}} \cdots p_{i_{t}}$ for arrows $p_{i_{1}}, \cdots, p_{i_{t}}$. Thus, $a_{1} D_{r, D_{p_{1}, p_{1}}(s)}+\cdots+$ $a_{\gamma_{1}} D_{r, D_{p_{1}, p_{1}}}(s)=\left(a_{i_{1}}+\cdots+a_{i_{t}}\right) D_{r, s}$. In summary,

$$
\left[D_{r, s}, D_{\mathfrak{c}}\right]=\left(a_{i_{0}}-a_{i_{1}}-\cdots-a_{i_{t}}\right) D_{r, s}
$$

which means that $D_{r, s}$ is the eigenvector under the adjoint action of $D_{\mathfrak{c}}$ with eigenvalue $-a_{i_{0}}+a_{i_{1}}+\cdots+a_{i_{r}}$. It follows that $\mathfrak{D}_{A L}=\mathbf{k} \mathfrak{B}_{A L}$ is a Lie ideal of $H H^{1}(\mathbf{k} \Gamma)$. We have proved the following result.

Theorem 5.7. (Structure Theorem of the First Hochschild Cohomology) Let $\Gamma$ be a connected planar finite acyclic quiver and let the ground field $\mathbf{k}$ be of characteristic 0 . Then the Lie algebra $H H^{1}(\mathbf{k} \Gamma)$ is the semi-direct sum of the Lie ideal $\mathfrak{D}_{A L}$ and the abelian Lie subalgebra $\mathfrak{D}_{F}$ :

$$
H H^{1}(\mathbf{k} \Gamma)=\mathfrak{D}_{A L} \rtimes_{\varphi} \mathfrak{D}_{F} .
$$

Here the action $\varphi$ of $\mathfrak{D}_{F}$ on $\mathfrak{D}_{A L}$ is given as follows. For each given $D_{\mathfrak{c}}$ with $\mathfrak{c}=$ $a_{1} D_{p_{1}, p_{1}}+a_{2} D_{p_{2}, p_{2}}+\cdots+a_{\gamma_{1}} D_{p_{\gamma_{1}}, p_{\gamma_{1}}}$ and $D_{r, s} \in \mathfrak{D}_{A L}$ with $r=p_{i_{0}}$ and $s=p_{i_{1}} \cdots p_{i_{t}}$, $D_{r, s}$ is the eigenvector under the adjoint action of $D_{\mathfrak{c}}$ with eigenvalue $-a_{i_{0}}+a_{i_{1}}+$ $\cdots+a_{i_{r}}$.

As noted in the introduction, the basis and semidirect sum decomposition of the first Hochschild cohomology in Theorem 5.5, Corollary 5.6 are Theorem 5.7, which are different from the one in [24]. 
From this proposition, we conclude that when two quivers are defined on the same unoriented graph, their outer differential Lie algebras are not isomorphic in general unless they have the same set of almost oriented cycles.

Acknowledgements. Li Guo acknowledges support by NSF grant DMS 1001855 and thanks the Center of Mathematical Sciences in Zhejiang University for hospitality. Fang Li takes this opportunity to express thanks to the support from the National Natural Science Foundation of China (No.11271318, No.11171296) and the Zhejiang Provincial Natural Science Foundation of China (No. LZ13A010001) and the Specialized Research Fund for the Doctoral Program of Higher Education of China (No. 20110101110010). This research was supported in part by the Project of Knowledge Innovation Program (PKIP) of Chinese Academy of Sciences, Grant No. KJCX2.YW.W10. The authors acknowledge the hospitality and support of the Kavli Institute for Theoretical Physics in China and the Morningside Center of Mathematics in Beijing in 2013. The authors thank the anonymous referee for helpful suggestions.

\section{REFERENCES}

[1] G. Ames, L. Cagliero, And P. Tirao, Comparison morphisms and the Hochschild cohomology ring of truncated quiver algebras, J. Algebra, 322 (2009), pp. 1466-1497.

[2] I. Assem, D. Simson, And A. Skowronski, Elements of the representation theory of associative algebras Vol I: Techniques of representation theory, London Mathematical Society Student Texts 65, Cambridge University Press, Cambridge, 2006.

[3] M. Auslander, I. Reiten, and S. O. Smal $\varnothing$, Representation theory of Artin algebra, Cambridge University Press, Cambridge, 1995.

[4] B. BollobÁs, Modern graph theory, Graduate Texts in Mathematics, 184 Springer-Verlag, 1998.

[5] P. J. Cassidy, L. Guo, W. F. Keigher, And W. Y. Sit, eds., Differential algebra and related topics, Proceedings of the International Workshop on Differential Algebra and Related Topics, Newark Campus of Rutgers, The State University of New Jersey, 2-3 November 2000. World Scientific, Singapore.

[6] C. CiBIs, Rigidity of truncated quiver algebras, Adv. Math., 79 (1990), pp. 18-42.

[7] W. Crawley-Boevey, Lectures on representations of quivers, http://www.amsta.leeds.ac. uk/ pmtwc/quivlecs.pdf.

[8] J. L. Gross and T. W. Tucker, Topological graph theory, John Wiley and Sons, New York, 1987.

[9] R. L. Grossman and R. G. Larson, Differential algebra structures on families of trees, Adv. Applied Math., 35 (2005), pp. 97-119.

[10] L. Guo And W. Keigher, On differential Rota-Baxter algebras, J. Pure Appl. Algebra, 212 (2008), pp. 522-540.

[11] D. HAPpeL, Hochschild cohomology of finite dimensional algebras, Lecture Notes in Math., Springer-Verlag, 1404 (1989), pp. 108-126.

[12] J. E. Humphreys, Introduction to Lie algebras and representation theory, GTM 9, SpringerVerlag, New York, 1972.

[13] E. Kolchin, Differential algebra and algebraic groups, Academic Press, New York, 1973.

[14] P. Lancaster and M. Tismenetsky, The theory of matrices with applications (Second Edition), Academic Press, London, 1985.

[15] F. LI, Characterization of left Artinian algebras through pseudo path algebras, J. Australia Math. Soc., 83 (2007), pp. 385-416.

[16] F. LI, Modulation and natural valued quiver of an algebra, Pacific J. Math., 256 (2012), pp. 105128.

[17] F. Li And L. L. Chen, The natural quiver of an artinian algebra, Algebras and Representation Theory, 13:5 (2010), pp. 623-636.

[18] F. Li AND Z. LiN, Approach to artinian algebras via natural quivers, Transactions of The American Mathematical Society, 364:3 (2012), pp. 1395-1411.

[19] A. LocAteli, Hochschild cohomology of truncated quiver algebras, Comm. Algebra, 27 (1999), pp. $645-664$. 
[20] J. A. Pena AND M. SAORIn, On the first Hochschild cohomology group of an algebra, Manscripta Math., 104 (2001), pp. 431-442.

[21] R. S. Pierce, Associative algebras, Graduate Texts in Mathematics, 88, Springer-Verlage, 1982.

[22] J. F. RitT, Differential equations from the algebraic standpoint, Amer. Math. Soc. Colloq. Publ., 14, Amer. Math. Soc., New York, 1932.

[23] S. SANChez-Flores, The Lie module structure on the Hochschild cohomology groups of monomial algebras with radical square zero, J. Algebra, 320 (2008), pp. 4249-4269.

[24] S. SANCHEZ-Flores, On the semisimplicity of the outer derivations of monomial algebras, Comm. Algebra, 39 (2011), pp. 3410-3434.

[25] M. Singer And M. van Der Put, Galois theory of linear differential equations, Springer, 2003.

[26] C. Strametz, The Lie algebra structure on the first Hochschild cohomology group of a monomial algebra, J. Algebra Appl., 5:3 (2006), pp. 245-270.

[27] W.-T. Wu, On the Decision Problem and the Mechanization of Theorem Proving in Elementary Geometry, Scientia Sinica, 21:2 (1978), pp. 159-172. Also reprinted in Contemporary Mathematics, 29 (1984), pp. 213-241.

[28] W.-T. Wu, A constructive theory of differential algebraic geometry based on works of J. F. Ritt with particular applications to mechanical theorem-proving of differential geometries, Differential Geometry and Differential Equations, (Shanghai, 1985). Lecture Notes in Math. 1255, Springer-Verlag, Berlin-New York, 1987, pp. 173-189.

[29] Y. G. XU, Y. HAN, AND W. F. JIANG, Hochschild cohomology of truncated quiver algebras, Science in China (Series A), 50:5 (2007), pp. 727-736. 